A. Szimayer, G. Dimitroff, S. Lorenz

A parsimonious multi-asset Heston model: calibration and derivative pricing 
(C) Fraunhofer-Institut für Techno- und Wirtschaftsmathematik ITWM 2009

ISSN 1434-9973

Bericht 171 (2009)

Alle Rechte vorbehalten. Ohne ausdrückliche schriftliche Genehmigung des Herausgebers ist es nicht gestattet, das Buch oder Teile daraus in irgendeiner Form durch Fotokopie, Mikrofilm oder andere Verfahren zu reproduzieren oder in eine für Maschinen, insbesondere Datenverarbeitungsanlagen, verwendbare Sprache zu übertragen. Dasselbe gilt für das Recht der öffentlichen Wiedergabe.

Warennamen werden ohne Gewährleistung der freien Verwendbarkeit benutzt.

Die Veröffentlichungen in der Berichtsreihe des Fraunhofer ITWM können bezogen werden über:

Fraunhofer-Institut für Techno- und Wirtschaftsmathematik ITWM Fraunhofer-Platz 1

67663 Kaiserslautern

Germany

Telefon: $\quad+49(0) 631 / 31600-0$

Telefax: $\quad+49(0) 631 / 31600-1099$

E-Mail: info@itwm.fraunhofer.de

Internet: www.itwm.fraunhofer.de 
Das Tätigkeitsfeld des Fraunhofer-Instituts für Techno- und Wirtschaftsmathematik ITWM umfasst anwendungsnahe Grundlagenforschung, angewandte Forschung sowie Beratung und kundenspezifische Lösungen auf allen Gebieten, die für Techno- und Wirtschaftsmathematik bedeutsam sind.

In der Reihe »Berichte des Fraunhofer ITWM « soll die Arbeit des Instituts kontinuierlich einer interessierten Öffentlichkeit in Industrie, Wirtschaft und Wissenschaft vorgestellt werden. Durch die enge Verzahnung mit dem Fachbereich Mathematik der Universität Kaiserslautern sowie durch zahlreiche Kooperationen mit internationalen Institutionen und Hochschulen in den Bereichen Ausbildung und Forschung ist ein großes Potenzial für Forschungsberichte vorhanden. In die Berichtreihe sollen sowohl hervorragende Diplom- und Projektarbeiten und Dissertationen als auch Forschungsberichte der Institutsmitarbeiter und Institutsgäste zu aktuellen Fragen der Techno- und Wirtschaftsmathematik aufgenommen werden.

Darüber hinaus bietet die Reihe ein Forum für die Berichterstattung über die zahlreichen Kooperationsprojekte des Instituts mit Partnern aus Industrie und Wirtschaft.

Berichterstattung heißt hier Dokumentation des Transfers aktueller Ergebnisse aus mathematischer Forschungs- und Entwicklungsarbeit in industrielle Anwendungen und Softwareprodukte - und umgekehrt, denn Probleme der Praxis generieren neue interessante mathematische Fragestellungen.

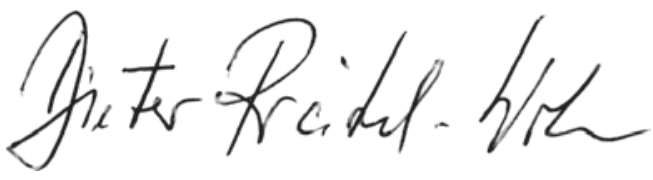

Prof. Dr. Dieter Prätzel-Wolters Institutsleiter

Kaiserslautern, im Juni 2001 



\title{
A Parsimonious Multi-Asset Heston Model: Calibration and Derivative Pricing
}

\author{
Alexander Szimayer* Georgi Dimitroff ${ }^{\dagger}$ Stefan Lorenz ${ }^{\dagger}$
}

\begin{abstract}
We present a parsimonious multi-asset Heston model. All single-asset submodels follow the well-known Heston dynamics and their parameters are typically calibrated on implied market volatilities. We focus on the calibration of the correlation structure between the single-asset marginals in the absence of sufficient liquid cross-asset option price data. The presented model is parsimonious in the sense that $d(d-1) / 2$ asset-asset cross-correlations are required for a $d$-asset Heston model. In order to calibrate the model, we present two general setups corresponding to relevant practical situations: (1) when the empirical cross-asset correlations in the risk neutral world are given by the user and we need to calibrate the correlations between the driving Brownian motions or (2) when they have to be estimated from the historical time series. The theoretical background, including the ergodicity of the multidimensional CIR process, for the proposed estimators is also studied.
\end{abstract}

Key Words: Heston model, multi-asset, option pricing, calibration, correlation

JEL Classification: G13

Mathematics Subject Classification (2000): 60G44, 60H30, 62M10

This version: July 16, 2009

*Institut für Gesellschafts- und Wirtschaftswissenschaften, Friedrich-Wilhelms-Universität Bonn

${ }^{\dagger}$ Fraunhofer-Institut für Techno- und Wirtschaftsmathematik Kaiserslautern 


\section{Introduction}

The Heston model is a derivative pricing framework widely used for financial markets including equity, commodity, and foreign exchange. The single-asset Heston is a twofactor model describing the dynamics of an asset and simultaneously the asset's volatility. In the traditional single-asset model, the parameters are calibrated from plain vanilla option price data, if available.

For valuing derivatives written on more than one asset, say $d$ assets, the Heston model can be extended accordingly to the multi-asset Heston model. For each asset and its corresponding volatility, the parameters can be calibrated in the same fashion as done for the single-asset Heston. However, the cross-correlations asset-asset, asset-volatility, and volatility-volatility cannot be calibrated from single-asset option price data. Note, here we use the expression cross-correlation if the factors belong to different single-asset Heston models.

In the absence of sufficient cross-asset option price data, we propose a method to fill in the missing cross-correlations. The method uses empirical correlations of the assets as input data. Hence, cross-correlations asset-volatility and volatility-volatility are not required as input data. The rationale is based on: (1) cross-correlations involving volatilities are hard to estimate from time series data since volatility is typically not observable and has to be filtered/constructed from market data (volatility implied by option prices or using proxies like volatility indices such as VDAX and VIX). (2) Taking the full set cross of correlations as input data restricts possible choices of asset-volatility correlations for each single-asset Heston model contained in the multi-asset framework. The correlation of an asset and the asset's volatility are not obtained empirically but from option price data. If the calibrated correlation does not satisfy the restrictions imposed by the empirical cross-correlations, the model breaks down.

Our method is based on the two-asset case, and can be extended to the full multi-asset model by considering all two-asset sub-models. Thus the stability of the method for $d$ assets is identical to the simple two-asset case, and the computer run times grow with $(d-1) d / 2$ (the number of two-dimensional sub-models). For the two-asset Heston, we 
compute the missing continuous time model parameters by a calibration approach. In the two-asset case there are four missing cross-correlations. The cross-correlation asset-asset is determined and the remaining three cross-correlations are expressed as functions of the other parameters. Paths are then generated using Monte-Carlo simulation. These paths are observed at the same frequency as the data on which the empirical correlation is based. From the simulated observations the simulated empirical correlation is computed. Using the bisection methodology, the model cross-correlation asset-asset is then adjusted such that the simulated empirical correlation and the empirical correlation coincide.

\section{Model}

In this section, the stochastic volatility model of Heston [1993] is reviewed. Subsequently, a parsimonious multi-asset extension is developed and studied for its properties.

\subsection{The Single-Asset Heston Model}

In the framework of Heston [1993], the risk-neutral dynamics of the asset price process $S(t)$ is given by

$$
\frac{\mathrm{d} S(t)}{S(t)}=(r(t)-q(t)) \mathrm{d} t+\sqrt{\nu(t)} \mathrm{d} W_{S}(t), \quad S(0)>0,
$$

where $r(t)$ is risk-free rate, $q(t)$ is the dividend rate, and $W_{S}(t)$ is a Wiener process. The volatility $\sqrt{\nu(t)}$ is a mean reverting process with risk-neutral dynamics

$$
\mathrm{d} \nu(t)=\kappa(\bar{\nu}-\nu(t)) \mathrm{d} t+\eta \sqrt{\nu(t)} \mathrm{d} W_{\nu}(t), \quad \nu(0)>0
$$

Thus, $\nu(t)$ describes a mean-reverting process with reversion rate $\kappa>0$, mean level $\bar{\nu}$, "volatility" $\eta>0$ and initial value $\nu(0)$. The process driving $\nu(t)$ is a Wiener process $W_{\nu}(t)$. To allow for possible leverage effects, $W_{S}(t)$ and $W_{\nu}(t)$ can be correlated with the coefficient $\rho_{S \nu}$, with $\left|\rho_{S \nu}\right|<1$. We can write $\mathrm{d} W_{S}(t) \mathrm{d} W_{\nu}(t)=\rho_{S \nu} \mathrm{d} t$. The parameters $\rho, \kappa, \bar{\nu}, \nu(0), \eta$ are assumed to be given, e.g., calibrated from plain vanilla option price data. 


\subsection{A Parsimonious Multi-Asset Heston Model}

Heston can be extended to a multi-asset model in different ways. For example, the Heston model can be extended by generalizing the real-valued variance rate process to a matrix valued version. Wishart processes represent the matrix analogue of the square-root meanreverting process and are used for volatility modeling by Gourieroux [2006].

We propose a parsimonious multi-asset extension of the single-asset Heston with properties: (1) each single-asset sub-model forms a traditional Heston model; and (2) parameters are single-asset Heston parameters and asset-asset cross-correlations. These properties are desirable since (1) enables using established calibration procedures for the single-asset sub-models parameters, and (2) is parsimonious in the sense that $d(d-1) / 2$ asset-asset cross-correlations are required for our $d$-asset Heston model.

Now consider a system of $d$ assets with price and volatility processes $\left(S_{1}(t), \nu_{1}(t)\right), \ldots$, $\left(S_{d}(t), \nu_{d}(t)\right)$. For $i=1, \ldots, d$ we can write (1) and (2) in the vectorized form

$$
\begin{aligned}
\left(\begin{array}{c}
d S_{i}(t) \\
d \nu_{i}^{2}(t)
\end{array}\right)= & \left(\begin{array}{c}
S_{i}(t)(r(t)-q(t)) \\
\kappa_{i}\left(\bar{\nu}_{i}-\nu_{i}(t)\right)
\end{array}\right) d t \\
& +\left(\begin{array}{cc}
S_{i}(t) \sqrt{\nu_{i}(t)} & 0 \\
0 & \eta_{i} \sqrt{\nu_{i}(t)}
\end{array}\right)\left(\begin{array}{cc}
1 & 0 \\
\rho_{i} & \sqrt{1-\rho_{i}^{2}}
\end{array}\right)\left(\begin{array}{c}
d W_{i}(t) \\
d \widetilde{W}_{i}(t)
\end{array}\right),
\end{aligned}
$$

where $W_{i}(t)$ and $\widetilde{W}_{i}(t)$ are independent Wiener processes. The parameter describing the bivariate process are collected in $\theta_{i}=\left(\rho_{i}, \kappa_{i}, \bar{\nu}_{i}, \nu_{i}^{2}(0), \eta_{i}\right)$.

The model is completely described except for the dependence structure. So far, the correlations asset-volatility are given for $i=1, \ldots, d$, but the cross-correlations asset-asset of $W_{i}(t)$ and $W_{j}(t)$, asset-volatility of $W_{i}(t)$ and $\widetilde{W}_{j}(t)$, and volatility-volatility of $\widetilde{W}_{i}(t)$ and $\widetilde{W}_{j}(t),(i \neq j)$, are not yet specified.

Define the $d$-dimensional Wiener processes $W(t)=\left(W_{1}(t), \ldots, W_{d}(t)\right)$ and $\widetilde{W}(t)=$ $\left(\widetilde{W}_{1}(t), \ldots, \widetilde{W}_{d}(t)\right)$. Our assumptions on the dependence structure are summarized below.

Assumption 2.1. The Wiener processes $W(t)$ and $\widetilde{W}(t)$ satisfy:

(1) $W(t)$ has correlation matrix $\Sigma^{S}=\left(\rho_{i, j}\right)_{1 \leq i, j \leq d}$, i.e., $d W_{i}(t) d W_{j}(t)=\rho_{i, j} d t ;^{1}$

\footnotetext{
${ }^{1} \mathrm{~A}$ matrix is a correlation matrix if and only if it is symmetric, positive semi-definite, and has ones
} 
(2) $\widetilde{W}(t)$ has correlation matrix $I_{d}$, i.e., $d \widetilde{W}_{i}(t) d \widetilde{W}_{j}(t)=\delta_{i, j} d t ;^{2}$

(3) $W(t)$ and $\widetilde{W}(t)$ are independent.

The dependence assumption on $(W(t), \widetilde{W}(t))$ is permissible. To see this, observe that the correlation matrix of $(W(t), \widetilde{W}(t))$ is

$$
\Sigma^{(W, \widetilde{W})}=\left(\begin{array}{cc}
\Sigma^{S} & 0 \\
0 & I_{d}
\end{array}\right) .
$$

Provided that $\Sigma^{S}$ is a correlation matrix, which holds by assumption, the matrix $\Sigma^{(W, \widetilde{W})}$ is also a correlation matrix.

Assumption (1) allows all possible correlation matrices for the asset prices. In contrast, assumptions (2) and (3) are restrictions stating that the dependence of the volatilities is carried via the correlations of the corresponding asset prices $\rho_{i, j}$, which is then transferred to each volatility by the corresponding Heston parameter asset-volatility correlation $\rho_{i}$ and $\rho_{j}$.

\subsubsection{Correlation Structure}

The model specification in (3) and Assumption 2.1 is implicit in the correlation structure of the vector process $\left(S_{1}(t), \nu_{1}^{2}(t), \ldots, S_{d}(t), \nu_{d}^{2}(t)\right)$. The correlation structure is stated in the following Proposition.

Proposition 2.2. Let $\left(S_{1}(t), \nu_{1}(t), \ldots, S_{d}(t), \nu_{d}(t)\right)$ be the 2d-dimensional process defined in (3) and Assumption 2.1 holds. Then for $1 \leq i, j \leq d$ the instantaneous covariances of the components are

(1) $\mathrm{d} S_{i}(t) \mathrm{d} S_{j}(t)=\rho_{i, j} S_{i}(t) S_{j}(t) \sqrt{\nu_{i}(t) \nu_{j}(t)} \mathrm{d} t ;$

(2) $\mathrm{d} S_{i}(t) \mathrm{d} \nu_{j}(t)=\rho_{i, j} \rho_{j} S_{i}(t) \eta_{j} \sqrt{\nu_{i}(t) \nu_{j}(t)} \mathrm{d} t$;

(3) $\mathrm{d} \nu_{i}(t) \mathrm{d} \nu_{j}(t)= \begin{cases}\rho_{i, j} \rho_{i} \rho_{j} \eta_{i} \eta_{j} \sqrt{\nu_{i}(t) \nu_{j}(t)} \mathrm{d} t, & \text { for } i \neq j, \\ \eta_{i} \eta_{j} \sqrt{\nu_{i}(t) \nu_{j}(t)} \mathrm{d} t, & \text { for } i=j .\end{cases}$

on the main diagonal.

${ }^{2} \delta_{i, j}$ is Kronecker's delta, i.e. $\delta_{i, j}=1$ if $i=j$, and $\delta_{i, j}=0$ if $i \neq j$. 
Thus the instantaneous correlations of the component are

$$
\begin{aligned}
& \text { (1) } \frac{\mathrm{d} S_{i}(t) \mathrm{d} S_{j}(t)}{\sqrt{\left(\mathrm{d} S_{i}(t)\right)^{2}\left(\mathrm{~d} S_{j}(t)\right)^{2}}}=\rho_{i, j} ; \\
& \text { (2) } \frac{\mathrm{d} S_{i}(t) \mathrm{d} \nu_{j}(t)}{\sqrt{\left(\mathrm{d} S_{i}(t)\right)^{2}\left(\mathrm{~d} \nu_{j}(t)\right)^{2}}}=\rho_{i, j} \rho_{j} ; \\
& \text { (3) } \frac{\mathrm{d} \nu_{i}(t) \mathrm{d} \nu_{j}(t)}{\sqrt{\left(\mathrm{d} \nu_{i}(t)\right)^{2}\left(\mathrm{~d} \nu_{j}(t)\right)^{2}}}= \begin{cases}\rho_{i, j} \rho_{i} \rho_{j}, & \text { for } i \neq j, \\
1, & \text { for } i=j .\end{cases}
\end{aligned}
$$

\subsubsection{Simulation Algorithm}

The multi-asset Heston model developed here can be simulated using the full truncated Euler method for example. For the time step $t \mapsto t+\Delta t$, the Euler scheme for single-asset Heston is given by

$$
\left(\begin{array}{c}
\log \widehat{S}(t+\Delta t) \\
\widehat{\nu}^{2}(t+\Delta t)
\end{array}\right)=\left(\begin{array}{c}
\log \widehat{S}(t)+(r(t)-q(t)) \Delta t+\sqrt{\widehat{\nu}(t) \Delta t} \varepsilon^{S} \\
\widehat{\nu}(t)+\kappa(\theta-\widehat{\nu}(t)) \Delta t+\eta \sqrt{\widehat{\nu}(t) \Delta t} \varepsilon^{\nu}
\end{array}\right)
$$

where $\left(\varepsilon^{S}, \varepsilon^{\nu}\right)$ is a bivariate standard normal random variable with zero mean and unit variances and correlation $\rho$. Equation (4) leaves aside numerical issues where the variance rate process may become negative. It can be dealt with using e.g. the full truncation scheme from Lord et al. [2008] or the Andersen approach (see Andersen [2007]).

For the multi-asset Heston the correlation structure set out in Assumption 2.1 suggests the following algorithm:

- simulate a normal random vector $\varepsilon^{S}=\left(\varepsilon_{1}^{S}, \ldots, \varepsilon_{d}^{S}\right)$ with covariance matrix $\Sigma^{S}$ by: (a) calculate the Cholesky square root $L$ of $\Sigma^{S}=\left(\rho_{i, j}\right)_{1 \leq i, j \leq d}$, i.e. $L$ is a lower triangular matrix with $L L^{\top}=\Sigma^{S}$;

(b) generate $d$ independent standard normally distributed random numbers $z_{1}, \ldots$, $z_{d}$, and set $z=\left(z_{1}, \ldots, z_{d}\right)^{\top}$

(c) compute $\varepsilon^{S}=L z$.

- simulate a normal random vector $\varepsilon^{\nu}=\left(\varepsilon_{1}^{\nu}, \ldots, \varepsilon_{d}^{\nu}\right)$ by:

(a) generate $d$ independent standard normally distributed random numbers $\widetilde{z}_{1}, \ldots$, 
$\widetilde{z}_{d}$, and set $\widetilde{z}=\left(\widetilde{z}_{1}, \ldots, \widetilde{z}_{d}\right)^{\top}$

(b) calculate $\varepsilon_{i}^{\nu}=\rho_{i} \varepsilon_{i}^{S}+\sqrt{1-\rho_{i}^{2}} \widetilde{z}_{i}$, for $i=1, \ldots, d$.

The algorithm follows essentially the construction implicit in Assumption 2.1. One can verify that the correlation matrix of $\left(\varepsilon^{S}, \varepsilon^{\nu}\right)$ corresponds to the one given in Proposition 2.2. The simulation algorithm can be applied to schemes other than Euler.

Computing the Cholesky square root of a symmetric positive semi-definite matrix of dimension $n \times n$ is a numerical problem of complexity $\mathcal{O}\left(n^{3}\right)$. Using the algorithm suggested here we apply this procedure to a $d \times d$-matrix. Alternatively, one could use a 'brute force' approach and compute the Cholesky decomposition of the correlation matrix given in Proposition 2.2 which is of dimension $2 d \times 2 d$. This would increase the complexity by factor 8 .

\subsection{Empirical Correlations}

Our model is designed for markets with little cross-asset option price data complicating the calibration of potential correlation parameters. Assuming that the single-asset Heston parameters are calibrated, the free parameters for correlating the single-asset Heston models are contained in the matrix $\Sigma^{S}$. Thus $(d-1) d / 2$ additional parameters have to be calibrated to correlate the single-asset models.

In the absence of sufficient cross-asset option price data, we rely on external estimates that we assume to be given as empirical correlations of the asset prices $\Sigma^{\mathrm{emp}}$. The key idea for calibration is to adjust the model cross-correlations in $\Sigma^{S}$ according to the observed empirical correlations $\Sigma^{\mathrm{emp}}$. It is important to note that $\Sigma^{S}$ is the correlation of infinitesimal noise carried by the Wiener process $W(t)$, whereas $\Sigma^{\text {emp }}$ is the correlation of observed discrete-time asset price data. This observation is crucial since the $\Sigma^{S}$ is not influenced by an equivalent measure transformations, while the cross-asset correlations may and typically will change. Therefore in one of the estimation setups considered below we will estimate the $\Sigma^{S}$ using $\Sigma^{\mathrm{emp}}$ under the historical measure. The switch to the physical measure under the Heston assumption for the shape of the market price of volatility risk is rather straightforward. 
Let us now formulate what is exactly meant by the empirical correlations $\Sigma^{\mathrm{emp}}$. For observation times $\left(t_{k}\right)_{k=0, \ldots, K}$ we are given asset price data $\left(S_{1}\left(t_{k}\right), \ldots, S_{d}\left(t_{k}\right)\right)_{k=0, \ldots, K}$. The asset prices are suitably transformed to returns, log-returns or first differences, i.e.: $X_{i}=$ $\left(X_{i}\left(t_{k}\right)\right)_{k=1, \ldots, K}$ with $X_{i}=F^{i}\left(S_{i}\right)$.

$\widehat{\Sigma}_{K}^{\mathrm{emp}}(\Sigma)$ is then the empirical correlation matrix of these $\left(X_{i}\right)_{i=1, \ldots, d}$ using the $K$-step time series.

In particular, the elements of $\widehat{\Sigma}_{K}^{\mathrm{emp}}(\Sigma)=\left(\widehat{\rho}_{i, j: K}^{\mathrm{emp}}(\Sigma)\right)_{1 \leq i, j \leq d}$ are defined by

$$
\widehat{\rho}_{i, j: K}^{\mathrm{emp}}(\Sigma)=\frac{\widehat{\nu}_{i, j: K}^{\mathrm{emp}}(\Sigma)}{\sqrt{\widehat{\nu}_{i, i: K}^{\mathrm{emp}}(\Sigma) \widehat{\nu}_{j, j: K}^{\mathrm{emp}}(\Sigma)}},
$$

where

$$
\widehat{\nu}_{i, j: K}^{\mathrm{emp}}(\Sigma)=\frac{1}{K-1} \sum_{k=1, \ldots, K}\left(X_{i}\left(t_{k}\right)-\bar{X}_{i}\right)\left(X_{j}\left(t_{k}\right)-\bar{X}_{j}\right), \quad \text { and } \quad \bar{X}_{i}=\frac{1}{K} \sum_{k=1, \ldots, K} X_{i}\left(t_{k}\right) .
$$

The transformation applied commonly to asset price data is to form log returns. Other alternatives are possible (see Example 1), though sensible transforms are closely related to scaled first differences.

Example 1. Transformations typically used include

(1) first differences: $\left.F\left(S\left(t_{0}\right), \ldots, S\left(t_{K}\right)\right)\right)=\left(S\left(t_{1}\right)-S\left(t_{0}\right), \ldots, S\left(t_{K}\right)-S\left(t_{K-1}\right)\right)$;

(2) returns: $\left.F\left(S\left(t_{0}\right), \ldots, S\left(t_{K}\right)\right)\right)=\left(\frac{S\left(t_{1}\right)-S\left(t_{0}\right)}{S\left(t_{0}\right)}, \ldots, \frac{S\left(t_{K}\right)-S\left(t_{K-1}\right)}{S\left(t_{K-1}\right)}\right)$;

(3) log-returns: $\left.F\left(S\left(t_{0}\right), \ldots, S\left(t_{K}\right)\right)\right)=\left(\log \left(S\left(t_{1}\right) / S\left(t_{0}\right)\right), \ldots, \log \left(S\left(t_{K}\right) / S\left(t_{K-1}\right)\right)\right)$.

In the following we will concentrate on the log-returns as the most widely used transforms of the time series. However, we must stress that the results will hold also for the returns and the first differences.

\subsubsection{Properties}

As already mentioned above we focus on the log-returns and for a discretized asset price $S\left(t_{k}\right)$ in the following we consider

$$
X\left(t_{k}\right):=\log \frac{S\left(t_{k}\right)}{S\left(t_{k-1}\right)} .
$$


Theorem 2.3. Let $\left(S_{i}(t), \nu_{i}(t)\right)_{i=1, \ldots, d}$ be the processes defined in (3) and Assumption 2.1 holds. Let $\pi_{n}=\left(t_{k, n}\right)_{k=1, \ldots, K_{n}(T)}$ be partitions of $[0, T]$, with $\operatorname{mesh}\left(\pi_{n}\right)=\max _{i=1, \ldots, K_{n}} \mid t_{i, n}-$ $t_{i-1, n} \mid \rightarrow 0$, for $n \rightarrow \infty$. Then for all $1 \leq i, j \leq d$ we have

(1) $\lim _{T \rightarrow 0} \lim _{n \rightarrow \infty} \widehat{\rho}_{i, j: K_{n}(T)}^{\mathrm{emp}}(\Sigma)=\rho_{i, j}$ almost surely

(2) $\lim _{T \rightarrow \infty} \lim _{n \rightarrow \infty} \hat{\rho}_{i, j: K_{n}(T)}^{\mathrm{emp}}(\Sigma)=\rho_{i, j} \frac{\mathbb{E} \sqrt{\nu_{i j}(\infty)}}{\sqrt{\mathbb{E} \nu_{i}(\infty) \mathbb{E} \nu_{j}(\infty)}}=\rho_{i, j} \frac{\mathbb{E} \sqrt{\nu_{i j}(\infty)}}{\sqrt{\bar{\nu}_{i} \bar{\nu}_{j}}}$ almost surely, where $\nu_{i}(\infty), \nu_{j}(\infty)$ and $\nu_{i j}(\infty)$ denote random variables having the stationary distributions of the processes $\left(\nu_{i}(t)\right),\left(\nu_{j}(t)\right)$ and $\left(\nu_{i}(t) \nu_{j}(t)\right)$ respectively.

Proof. A straightforward transformation of the expression for $\widehat{\rho}_{i, j: K_{n}(T)}^{\mathrm{emp}}(\Sigma)$ yields

$$
\widehat{\rho}_{i, j: K_{n}(T)}^{\operatorname{minp}}(\Sigma)=\frac{\sum_{k=1}^{K_{n}(T)} X_{i}\left(t_{k}\right) X_{j}\left(t_{k}\right)-K_{n}(T) \bar{X}_{i} \bar{X}_{j}}{\sqrt{\sum_{k=1}^{K_{n}(T)} X_{i}^{2}\left(t_{k}\right)-K_{n}(T) \bar{X}_{i}^{2}} \sqrt{\sum_{k=1}^{K_{n}(T)} X_{j}^{2}\left(t_{k}\right)-K_{n}(T) \bar{X}_{j}^{2}}} .
$$

Following the model (3) we have

$$
S_{i}(t)=S_{i}(0) \exp \left[\int_{0}^{t} \sqrt{\nu_{i}(s)} \mathrm{d} W_{i}(s)+\int_{0}^{t}\left(r_{i}(s)-q_{i}(s)\right) \mathrm{d} s-\frac{1}{2} \int_{0}^{t} \nu_{i}(s) \mathrm{d} s\right]
$$

and the very definition of quadratic variation implies that almost surely the following convergence holds:

$$
\begin{aligned}
\sum_{k=1}^{K_{n}(T)} X_{i}\left(t_{k}\right) X_{j}\left(t_{k}\right) & =\sum_{k=1}^{K_{n}(T)}\left(\log S_{i}\left(t_{k}\right)-\log S_{i}\left(t_{k-1}\right)\left(\log S_{j}\left(t_{k}\right)-\log S_{j}\left(t_{k-1}\right)\right.\right. \\
& \underset{n \rightarrow \infty}{\longrightarrow}\left\langle\log S_{i}, \log S_{j}\right\rangle_{T}=\rho_{i, j} \int_{0}^{t} \sqrt{\nu_{i}(s) \nu_{j}(s)} \mathrm{d} s .
\end{aligned}
$$

On the other hand we have

$$
K_{n}(T) \bar{X}_{i} \bar{X}_{j}=\frac{1}{K_{n}(T)} \log \frac{S_{i}(T)}{S_{i}(0)} \log \frac{S_{j}(T)}{S_{j}(0)} \underset{n \rightarrow \infty}{\longrightarrow} 0, \quad \mathbb{P}-\text { a.s. }
$$

Combining (7), (8) and (9) we obtain

$$
\lim _{n \rightarrow \infty} \widehat{\rho}_{i, j: K_{n}(T)}^{\mathrm{emp}}(\Sigma)=\rho_{i, j} \frac{\int_{0}^{T} \sqrt{\nu_{i}(s) \nu_{j}(s)} \mathrm{d} s}{\sqrt{\int_{0}^{T} \nu_{i}(s) \mathrm{d} s} \sqrt{\int_{0}^{T} \nu_{j}(s) \mathrm{d} s}} .
$$


Letting $T \rightarrow 0$ in (10) and recalling the continuity of $\nu_{i}$ we obtain the first claim in the theorem.

For the second claim we need the ergodicity of the processes $\nu_{i}, \nu_{j}$ and $\left(\nu_{i}, \nu_{j}\right)$. This is well-known for the one dimensional CIR process. In the Appendix we provide the ergodicity of $\left(\nu_{i}, \nu_{j}\right)$ as we were not able to find a reference for this fact in the existing literature. Clearly, the two dimensional result implies the ergodicity of the one dimensional marginals. We use the approach as described in Has'minskii [1980]. Once the ergodicity of the above processes is verified, we can apply the ergodic theorem to obtain the following almost sure convergence:

$$
\lim _{n \rightarrow \infty} \widehat{\rho}_{i, j: K_{n}(T)}^{\mathrm{emp}}(\Sigma)=\rho_{i, j} \frac{\frac{1}{T} \int_{0}^{T} \sqrt{\nu_{i}(s) \nu_{2}(s)} \mathrm{d} s}{\sqrt{\frac{1}{T} \int_{0}^{T} \nu_{i}(s) \mathrm{d} s} \sqrt{\frac{1}{T} \int_{0}^{T} \nu_{2}(s) \mathrm{d} s}} \stackrel{T \rightarrow \infty}{\longrightarrow} \rho_{i, j} \frac{\mathbb{E} \sqrt{\nu_{i}(\infty) \nu_{2}(\infty)}}{\sqrt{\mathbb{E} \nu_{i}(\infty)} \sqrt{\mathbb{E} \nu_{2}(\infty)}} .
$$

Clearly the expectation of the invariant distribution of $\nu_{i}(t)$ is the mean reversion level $\bar{\nu}_{i}$ for all $i=1, \ldots, d$. This completes the proof.

\section{Remark 2.4.}

(1) The first part of Theorem 2.3 states that calculating correlations from high-frequency data observed over a rather short period renders $\widehat{\Sigma}^{\mathrm{emp}}(\Sigma)=\Sigma$. Thus $\Sigma=\Sigma^{\mathrm{emp}}$, and the correlation matrix is therefore not adjusted.

(2) Note, that the first part of Theorem 2.3 is a theoretical statement. High-frequency data -if available at all-is typically subject to other effects, e.g., caused by the market micro-structure. These effects are making the observations noisy and are potentially distorting the correlation estimates. Therefore, we adopt the second statement as our approach for estimating the correlation structure.

\section{Remark 2.5.}

(1) The Cauchy-Schwarz inequality immediately gives $\left|\widehat{\rho}_{i, j ; n}^{\mathrm{emp}}(\Sigma)\right| \leq\left|\rho_{i, j}\right|$ asymptotically for big $n$.

(2) The empirical correlation is approximately linear in $\rho$ with a positive slope strictly smaller than 1. Varying the model correlation $\rho_{i, j}$ by $\Delta \rho$, we can write

$$
\widehat{\rho}_{i, j ; n}^{\mathrm{emp}}(\Sigma+\Delta \rho) \approx \widehat{\rho}_{i, j ; n}^{\mathrm{emp}}(\Sigma)+\Delta \rho \frac{\mathbb{E} \sqrt{\nu_{i}(s) \nu_{j}(\infty)}}{\sqrt{\mathbb{E} \nu_{i}(\infty)} \sqrt{\mathbb{E} \nu_{j}(\infty)}} .
$$


where the distribution of $\left(\nu_{1}(\infty), \ldots, \nu_{d}(\infty)\right)$ depends on $\Sigma$.

(3) The range of the possible empirical correlations is strictly smaller than $[-1,1]$, except for the case if two single-asset Heston sub-models are perfectly correlated in the asset price and the variance rate process, which we want to exclude as it introduces singularity into the model.

\section{Overview of the proposed calibration procedures}

We are given the parameters $\theta_{i}=\left(\rho_{i}, \kappa_{i}, \bar{\nu}_{i}^{2}, \nu_{i}^{2}(0), \eta_{i}\right)$ for each single-asset Heston model obtained by single-asset Heston calibration routines. These parameters describe the dynamics of the model under the equivalent martingale measure. It remains to calibrate the correlation structure between the one-dimensional models. The ideal situation would be if we had a liquid market of correlation sensitive cross-asset derivatives which can be used for calibration. Unfortunately, the existence of such markets is rather an exception and therefore we focus on the alternative to use the historical asset time series to determine the correlations, which is done by calculating the historical empirical cross-asset correlations and extracting the $\Sigma$ out of them via passing to the physical measure. Observe that $\Sigma$ is invariant under equivalent measure change. The input of the algorithm are the historical time series of the assets.

In some situations it is sensible to assume that the cross-asset empirical correlations under the equivalent martingale measure are provided and our task is to calibrate the infinitesimal correlations $\Sigma$ directly under the relevant equivalent martingale measure. In this case we have as an input the expected empirical correlations.

In the second setup, calibrating the yet unknown parameters contained in the correlation matrix $\Sigma^{S}$ is then solving the problem

$$
\min _{\Sigma \in \operatorname{Cor}(d)}\left\|\mathbb{E} \widehat{\Sigma}^{\mathrm{emp}}(\Sigma)-\Sigma^{\mathrm{emp}}\right\|
$$

where $\operatorname{Cor}(d)$ is the space of $d \times d$-dimensional correlation matrices, i.e., $d \times d$-matrices over $\mathbb{R}$ that are symmetric, positive semi-definite and unit elements on the main diagonal, and $\|\cdot\|$ is a suitable matrix-norm. 


\section{Calibration under the equivalent martingale mea- sure}

We first start by describing the algorithm in the setup where we assume the cross-asset empirical correlations under the equivalent martingale measure are provided and we aim to determine $\Sigma$. We call the algorithm calibration with correlation adjustment. The first setup, where we extract the empirical correlations from the historical data is provided in section 5 .

\subsection{Correlation adjustment algorithm}

The input is a $d \times d$-dimensional matrix $\Sigma^{\mathrm{emp}}$, given by the user, which does not necessarily have to be positive semi-definite. It only has to be symmetric with diagonal entries of value 1 . This is then taken as the expected empirical correlation matrix under the equivalent martingale measure. From section 2.2 we know, that we do not need to solve a $d$-dimensional problem, but $d(d-1) / 2$ 2-dimensional problems. For these 2-dimensional problems, we propose an algorithm that calibrates the model and returns the desired correlation matrix $\Sigma^{s}$.

First, the two-asset model and its calibration is investigated. With given Heston parameters $\theta_{1}$ and $\theta_{2}$ for the two models, we have $\mathbb{E} \widehat{\rho}_{1,2}^{\mathrm{emp}}\left(\rho_{1,2}\right)=f_{\left(\theta_{1}, \theta_{2}\right)}\left(\rho_{1,2}\right)$, for a strictly increasing and continuous function $f_{\left(\theta_{1}, \theta_{2}\right)}:[-1,1] \mapsto \mathbb{R}$, parameterized by $\theta_{1}$ and $\theta_{2}$. Given that $\rho_{1,2}^{\mathrm{emp}}$ is contained in the range $\left\{f_{\left(\theta_{1}, \theta_{2}\right)}(x):|x| \leq 1\right\}$, the model can be uniquely calibrated by numerically solving the equation $\mathbb{E} \widehat{\rho}_{1,2}^{\mathrm{emp}}\left(\rho_{1,2}\right)=\rho_{1,2}^{\mathrm{emp}} \cdot 3$

\footnotetext{
${ }^{3}$ Remember that here $\rho_{1,2}^{\mathrm{emp}}$ is provided by the user.
} 


\subsubsection{Two-Asset Calibration}

Setting $d=2$, we can expand (3) to the four-dimensional system:

$$
\begin{aligned}
& \left(\begin{array}{c}
d S_{1}(t) \\
d \nu_{1}^{2}(t) \\
d S_{2}(t) \\
d \nu_{2}^{2}(t)
\end{array}\right)=\left(\begin{array}{c}
S_{1}(t)(r(t)-q(t)) \\
\kappa_{1}\left(\bar{\nu}_{1}^{2}-\nu_{1}^{2}(t)\right) \\
S_{2}(t)(r(t)-q(t)) \\
\kappa_{2}\left(\bar{\nu}_{2}^{2}-\nu_{2}^{2}(t)\right)
\end{array}\right) d t \\
& +\operatorname{diag}\left(\begin{array}{c}
S_{1}(t) \nu_{1}(t) \\
\eta_{1} \sqrt{\nu_{1}^{2}(t)} \\
S_{2}(t) \nu_{2}(t) \\
\eta_{2} \sqrt{\nu_{2}^{2}(t)}
\end{array}\right)\left(\begin{array}{cccc}
1 & 0 & 0 & 0 \\
\rho_{1} & \sqrt{1-\rho_{1}^{2}} & 0 & 0 \\
0 & 0 & 1 & 0 \\
0 & 0 & \rho_{2} & \sqrt{1-\rho_{2}^{2}}
\end{array}\right)\left(\begin{array}{c}
d W_{1}(t) \\
d \widetilde{W}_{1}(t) \\
d W_{2}(t) \\
d \widetilde{W}_{2}(t)
\end{array}\right)
\end{aligned}
$$

where $W_{1}(t)$ and $W_{2}(t)$ are correlated with $\rho_{1,2}$, and the pairs $W_{1}(t)$ and $\widetilde{W}_{1}(t), W_{2}(t)$ and $\widetilde{W}_{2}(t)$ and $\widetilde{W}_{1}(t)$ and $\widetilde{W}_{2}(t)$ are independent.

For $\left|\rho_{1,2}\right|<1$ we apply the standard Gram-Schmidt-type orthogonalization procedure and define $\bar{W}_{1}(t)=W_{1}(t)$ and $\bar{W}_{2}(t)=\left(\rho_{1,2} W_{1}(t)-W_{2}(t)\right) / \sqrt{1-\rho_{1,2}^{2}} \cdot{ }^{4}$ Then $\left(\bar{W}_{1}(t), \widetilde{W}_{1}(t), \bar{W}_{1}(t), \widetilde{W}_{2}(t)\right)$ is a Wiener process with uncorrelated components. The latter part of (13) then becomes

$$
\underbrace{\left(\begin{array}{cccc}
1 & 0 & 0 & 0 \\
\rho_{1} & \sqrt{1-\rho_{1}^{2}} & 0 & 0 \\
\rho_{1,2} & 0 & \sqrt{1-\rho_{1,2}^{2}} & 0 \\
\rho_{2} \rho_{1,2} & 0 & \rho_{2} \sqrt{1-\rho_{1,2}^{2}} & \sqrt{1-\rho_{2}^{2}}
\end{array}\right)}_{=: L}\left(\begin{array}{c}
d \bar{W}_{1}(t) \\
d \widetilde{W}_{1}(t) \\
d \bar{W}_{2}(t) \\
d \widetilde{W}_{2}(t)
\end{array}\right) .
$$

The correlation matrix is then given by

$$
C=L L^{\top}=\left(\begin{array}{cccc}
1 & \rho_{1} & \rho_{1,2} & \rho_{1,2} \rho_{2} \\
\rho_{1} & 1 & \rho_{1,2} \rho_{1} & \rho_{1,2} \rho_{1} \rho_{2} \\
\rho_{1,2} & \rho_{1,2} \rho_{1} & 1 & \rho_{2} \\
\rho_{1,2} \rho_{2} & \rho_{1,2} \rho_{1} \rho_{2} & \rho_{2} & 1
\end{array}\right)
$$

\footnotetext{
${ }^{4}$ Note that for $\left|\rho_{1,2}\right|=1$ we have the linear dependence $W_{1}(t)=\rho_{1,2} W_{2}(t)$, and $W_{2}(t)$ and its transform $\bar{W}_{2}(t)$ drop effectively out of the system.
} 
We see that the cross-correlation over the two distinct asset-volatility sub-models is specified by the asset-volatility correlations $\rho_{1}$ and $\rho_{2}$ of the single-asset Heston models, and the asset-asset cross-correlation $\rho_{1,2}$, i.e.

$$
C^{(1,2)}=\rho_{1,2}\left(\begin{array}{cc}
1 & \rho_{2} \\
\rho_{1} & \rho_{1} \rho_{2}
\end{array}\right) .
$$

Given the single-asset parameters, the dependence structure can be expressed exclusively by a single parameter, the asset-asset cross-correlation $\rho_{1,2}$.

Remark 4.1. Let $\left(X_{i}, Y_{i}\right)_{i=1, \ldots, K}$ be a sequence of independent identically distributed random variables, where $\left(X_{i}, Y_{i}\right)$ follows a bivariate normal distribution with correlation $\rho$. Then the correlation estimator

$$
\widehat{\rho}_{K}=\frac{\sum_{i=1}^{K}\left(X_{i}-\bar{X}_{K}\right)\left(Y_{i}-\bar{Y}_{K}\right)}{\sqrt{\sum_{i=1}^{K}\left(X_{i}-\bar{X}_{K}\right)^{2} \sum_{i=1}^{K}\left(Y_{i}-\bar{Y}_{K}\right)^{2}}}
$$

is consistent, i.e, $\widehat{\rho}_{K} \rightarrow \rho$ almost surely, for $K \rightarrow \infty$, and the variance is

$$
\operatorname{var}\left(\widehat{\rho}_{K}\right)=\frac{1-\rho^{2}}{K-2}
$$

Next, we have to numerically find the values $\rho$ which solve the problem

$$
\min _{|\rho| \leq 1}\left|\mathbb{E} \widehat{\rho}_{i, j}^{\mathrm{emp}}(\rho)-\rho^{\mathrm{emp}}\right|
$$

This can simply be done by some line search method such as bisectioning. Such a bisection procedure adapted to the given problem is stated here:

Input: Lower bound $\underline{\rho}=-1$, upper bound $\bar{\rho}=1,{ }^{5}$ accuracy $\epsilon$

Algorithm: (1) Set $\rho_{i, j}^{\mathrm{empA}}=\underline{\rho}$ and $\rho_{i, j}^{\mathrm{empB}}=\bar{\rho}=1$

(2) Define $\underline{\mathbb{E}}:=\mathbb{E}\left(\widehat{\rho}_{i, j}^{\mathrm{emp}}(\underline{\rho})\right)$ and $\overline{\mathbb{E}}:=\mathbb{E}\left(\hat{\rho}_{i, j}^{\mathrm{emp}}(\bar{\rho})\right)$ and define $\Delta \underline{\mathbb{E}}:=\underline{\mathbb{E}}-\rho_{i, j}^{\mathrm{emp}}$.

(3) Update parameters:

$$
\begin{aligned}
\rho_{i, j}^{\mathrm{empNew}} & :=0.5 * \rho_{i, j}^{\mathrm{empA}}-\rho_{i, j}^{\mathrm{empB}} \\
\mathbb{E}^{*} & :=\mathbb{E}\left(\widehat{\rho}_{i, j}^{\mathrm{emp}}\left(\rho_{i, j}^{\mathrm{empNew}}\right)\right) \\
\Delta \mathbb{E} & :=\mathbb{E}^{*}-\rho_{i, j}^{\mathrm{emp}} .
\end{aligned}
$$

\footnotetext{
${ }^{5}$ The choice of these bounds is reasonable, as we are looking for correlations.
} 
(4) If $\Delta \mathbb{E}<\epsilon \rightarrow$ STOP and output $\rho_{i, j}^{\mathrm{empNew}}$.

If $(\Delta \underline{\mathbb{E}} \cdot \Delta \mathbb{E})<0 \rightarrow \rho_{i, j}^{\mathrm{empB}}=\rho_{i, j}^{\mathrm{empNew}}$ and GOTO step 3.

If $(\Delta \underline{\mathbb{E}} \cdot \Delta \mathbb{E})>0 \rightarrow \rho_{i, j}^{\mathrm{empA}}=\rho_{i, j}^{\mathrm{empNew}}$ and GOTO step 3 .

Output: Optimal parameter $\rho_{i, j}^{\mathrm{empNew}}$.

Throughout the whole procedure the calculation of $\mathbb{E} \hat{\rho}_{i, j}^{\mathrm{emp}}(\rho)$ is needed. This can be done by simple Monte-Carlo simulation as demonstrated in the following algorithm:

Input: Number of Monte-Carlo simulations $N$ and $N$ simulated paths $\left(P_{i}^{1}, P_{i}^{2}\right), 1 \leq i \leq$ $N .^{6}$

Algorithm: (1) Define $S:=0, i:=1$

(2) Take paths $P_{i}^{1}$ and $P_{i}^{2}$ and calculate their correlation $\rho_{P}$ using one of the transformation methods described in Example 1.

(3) Update $S=S+\rho_{P}$ and $i=i+1$.

If $i<N$ GOTO step 2.

(4) Calculate the mean value $M:=S / N$

Output: Monte-Carlo estimate $M$.

Remark 4.2. Observe that the $\widehat{\rho}_{i, j}^{\mathrm{emp}}(\rho)$ is actually a ratio of time averages and therefore the ergodic theorem states that it will converge to its expected value for large $T$. Therefore for calculating the expectation it is enough to take a fairly small number of Monte-Carlo runs. In our tests a value of 10 already provided good results.

This produces a symmetric matrix $\Sigma=\left(\rho_{i, j}\right)_{1 \leq i, j \leq d}$, which is our candidate $\Sigma^{\star}$ to solve equation (11).

The calibration is completed if the symmetric matrix $\Sigma$ is a correlation matrix, i.e. $\Sigma \in \operatorname{Cor}(d)$, as required in (11). If $\Sigma$ is not a correlation matrix, we propose three ways of transforming $\Sigma$ such that the transform is in $\operatorname{Cor}(d)$.

\footnotetext{
${ }^{6}$ Simulation of these paths can be done by Euler discretization possibly using variance reduction methods such as antithetic paths. Note that the paths are simulated with a correlation of $\rho$.
} 


\subsubsection{Multi-Asset Calibration}

In the general multi-asset setting, the two-asset procedure can be carried out for all asset pairs. The correlation matrix has two kinds of blocks

$$
C^{(i)}=\left(\begin{array}{cc}
1 & \rho_{i} \\
\rho_{i} & 1
\end{array}\right), \quad \text { and } \quad C^{(i, j)}=\rho_{i, j}\left(\begin{array}{cc}
1 & \rho_{j} \\
\rho_{i} & \rho_{i} \rho_{j}
\end{array}\right) \text {. }
$$

Using these blocks, the whole correlation matrix can be respresented. For example $C$ in (14) can be expressed as

$$
C=L L^{\top}=\left(\begin{array}{cc}
C^{(1)} & C^{(1,2)} \\
C^{(2,1)} & C^{(2)}
\end{array}\right)
$$

By this block representation, we can rearrange the single blocks, such that for any two assets $i$ and $k$ we obtain a matrix

$$
C_{i, k}=L_{i, k} L_{i, k}^{\top}=\left(\begin{array}{cc}
C^{(i)} & C^{(i, k)} \\
C^{(k, i)} & C^{(k)}
\end{array}\right) .
$$

After we have repeated the bisection procedure for all 2-dimensional problems, we get a candidate $\Sigma^{\star}$ to solve equation (11). To validate this candidate $\Sigma^{\star}$, we must check whether $\Sigma^{\star}$ is positive semi-definite or not. If the test succeeds, the calibration is finished. Otherwise, some regularization has to be done (see section 4.2).

\subsection{Generate a valid correlation matrix}

We investigated three ways of transforming $\Sigma^{\star}$ such that the resulting matrix is in $\operatorname{Cor}(d)$.

\subsubsection{Regularization by Jäckel [2002]}

One way out of this problem is a regularization proposed by Jäckel [2002]. The following algorithm briefly demonstrates how this regularization works:

Input: Model correlation matrix $\Sigma^{\star}$ (not necessarily positive definite).

Algorithm: (1) Do an eigenvalue decomposition of $\Sigma^{\star} \rightarrow \Sigma^{\star}=S \Lambda S^{T}$, with $\Lambda=$ $\operatorname{diag}\left(\lambda_{i}\right)$. Here, $\lambda_{i}, i=1, \ldots, d$ represents the eigenvalues of $\Sigma^{\star}$. 
(2) Define the diagonal matrix $\Lambda^{\prime}$ with $\lambda_{i}^{\prime}= \begin{cases}\lambda_{i}: & \lambda_{i} \geq 0 \\ 0: & \lambda_{i}<0\end{cases}$

(3) Create the diagonal matrix $T$ with $t_{i}:=\left[\sum_{m} s_{i m}^{2} \lambda_{m}^{\prime}\right]^{-1}$.

(4) Define $B:=\sqrt{T} S \sqrt{\Lambda^{\prime}}$.

(5) $\widehat{\Sigma^{\star}}:=B B^{T}$

Output: Positive definite correlation matrix $\widehat{\Sigma}^{\star}$.

\subsubsection{Regularization by Mishra [2004]}

Another way out of the problem of non-positive definite correlation matrices is a regularization proposed by Mishra [2004]. The following algorithm briefly demonstrates how this regularization works:

Input: Model correlation matrix $\Sigma^{\star}$ (not necessarily positive definite).

Algorithm: (1) Do an eigenvalue decomposition of $\Sigma^{\star} \rightarrow \Sigma^{\star}=S \Lambda S^{T}$, with $\Lambda=$ $\operatorname{diag}\left(\lambda_{i}\right)$. Here, $\lambda_{i}, i=1, \ldots, d$ represents the eigenvalues of $\Sigma^{\star}$.

(2) Define the diagonal matrix $\Lambda^{\prime}$ with $\lambda_{i}^{\prime}= \begin{cases}\lambda_{i}: & \lambda_{i} \geq 0 \\ 0: & \lambda_{i}<0\end{cases}$

(3) Generate $d$ uniformly $(0,1)$-distributed random numbers and add them to the diagonal elements of $\Lambda$. Normalize $\Lambda$ such that its trace is equal to $d$.

(4) By random walk methods of optimization find best possible $\Lambda$ such that $\operatorname{trace}(\lambda)=m$ and $\widehat{\Sigma}^{\star}=S \Lambda S^{T}$ has positive determinant and is positive definite and closest (wrt. maximum norm) to $\Sigma^{\star}$.

(5) IF all diagonal entries of $\widehat{\Sigma}^{\star}$ are approximately 1 , depending on a tolerance level THEN STOP, ELSE set diagonal entries to 1 and GOTO step 1.

Output: Positive definite correlation matrix $\widehat{\Sigma}^{\star}$.

Remark 4.3. By construction, both algorithms are well defined, as they both deliver a positive definite correlation matrix, if they stop. Obviously, the first two steps of Jaeckel's and 
Mishra's algorithms are equal. In the proceeding steps, Jaeckel uses a rather deterministic way to create the positive definite correlation matrix, while Mishra uses a stochastic algorithm. Unfortunately, there is also a chance of non-convergence for the Mishra Algorithm in a case that the intermediate matrix $\widehat{\Sigma}^{\star}$ in the intermediate step 4 is near-singular.

\subsubsection{Regularization by convex combination}

If the input matrix $\Sigma^{\mathrm{emp}}$ is already positive definite, but the model matrix $\Sigma^{\star}$ is not, there is another easy way of creating a positive definite correlation matrix as a convex combination of the original input matrix and the model matrix. The following algorithm briefly demonstrates how this regularization works.

Input: Correlation matrix $\Sigma$ (positive definite) and model correlation matrix $\Sigma^{\star}$ (not necessarily positive definite).

Algorithm: By standard line search algorithms find smallest $\lambda \in[0,1]$ such that $\widehat{\Sigma}^{\star}:=$ $\lambda \Sigma+(1-\lambda) \Sigma^{\star}$ is positive definite.

Output: Positive definite correlation matrix $\widehat{\Sigma}^{\star}$

Remark 4.4. By construction, the matrix $\widehat{\Sigma}^{\star}$ is symmetric and positive definite and its diagonal contains only values 1 , and is therefore a valid correlation matrix.

\subsubsection{Comparison of the different methods}

We tested the regularization procedure of all three methods for one example. As the convex combination only works if the empirical input matrix is already a correlation matrix, we constructed the example such that this is fulfilled. We chose 10 assets with correlation

$$
\rho_{i j}= \begin{cases}0.8 & \text { if } \quad i \neq j \\ 1 & \text { if } \quad i=j\end{cases}
$$




\begin{tabular}{llll}
\hline Norm & Jäckel & Mishra & Convex \\
\hline Euclidean norm & $5.66 \%$ & $8.27 \%$ & $37.63 \%$ \\
maximum norm & $1.03 \%$ & $1.15 \%$ & $5.25 \%$ \\
row-sum norm & $8.50 \%$ & $10.00 \%$ & $39.13 \%$ \\
\hline
\end{tabular}

Table 1: Errors of different methods

except for one asset pair (asset 1 and asset 8), for which we defined a correlation of 0.56. This matrix is a valid correlation matrix. The calibrated matrix $\Sigma^{\star}$ is in this setting

$$
\left(\begin{array}{cccccccccc}
100.00 \% & 84.61 \% & 84.61 \% & 83.87 \% & 84.61 \% & 84.61 \% & 84.61 \% & 84.61 \% & 61.60 \% & 84.61 \% \\
84.61 \% & 100.00 \% & 83.13 \% & 83.13 \% & 83.87 \% & 83.87 \% & 83.13 \% & 83.87 \% & 83.87 \% & 83.13 \% \\
84.61 \% & 83.13 \% & 100.00 \% & 83.13 \% & 83.87 \% & 83.87 \% & 83.87 \% & 83.87 \% & 83.87 \% & 83.13 \% \\
83.87 \% & 83.13 \% & 83.13 \% & 100.00 \% & 83.13 \% & 83.13 \% & 83.13 \% & 83.87 \% & 83.87 \% & 83.13 \% \\
84.61 \% & 83.87 \% & 83.87 \% & 83.13 \% & 100.00 \% & 84.61 \% & 84.61 \% & 85.35 \% & 85.35 \% & 85.35 \% \\
84.61 \% & 83.87 \% & 83.87 \% & 83.13 \% & 84.61 \% & 100.00 \% & 84.61 \% & 85.35 \% & 84.61 \% & 84.61 \% \\
84.61 \% & 83.13 \% & 83.87 \% & 83.13 \% & 84.61 \% & 84.61 \% & 100.00 \% & 84.61 \% & 84.61 \% & 84.61 \% \\
84.61 \% & 83.87 \% & 83.87 \% & 83.87 \% & 85.35 \% & 85.35 \% & 84.61 \% & 100.00 \% & 84.61 \% & 84.61 \% \\
61.60 \% & 83.87 \% & 83.87 \% & 83.87 \% & 85.35 \% & 84.61 \% & 84.61 \% & 84.61 \% & 100.00 \% & 84.61 \% \\
84.61 \% & 83.13 \% & 83.13 \% & 83.13 \% & 85.35 \% & 84.61 \% & 84.61 \% & 84.61 \% & 84.61 \% & 100.00 \%
\end{array}\right)
$$

This matrix has negative eigenvalues and therefore it is not positive semi-definite. After trying the different regularizations we can compute the error in terms of some matrix norm. According to different norms, the errors can be seen in table 1. For the chosen setting, Jäckel clearly performs best. Mishra also produces good results. Although convex combination is the easiest method, it returns the worst results.

To do further investigations, we also calculated an error matrix $\Sigma_{\text {error }}:=\left|\widehat{\Sigma}^{\star}-\Sigma^{\star}\right|$. Figures 1 to 3 graphically show the distribution of these errors in the error matrices for the different methods. To illustrate the differences of the three methods, we applied the same scale to every graph. In figure 1 one clearly sees, that the error is concentrated vertically and horizontally from the critical value, which was the correlation of asset 1 and asset 9. Using Mishra's method, the errors in figure 2 are distributed more equally all over the error matrix, however, the maximum error is worse than for Jäckel. What we can already derive from the numbers in table 1 gets even more obvious when we take a look at figure 3 for the case of convex combination. This method almost completely fails and there are large errors all over the whole matrix. 


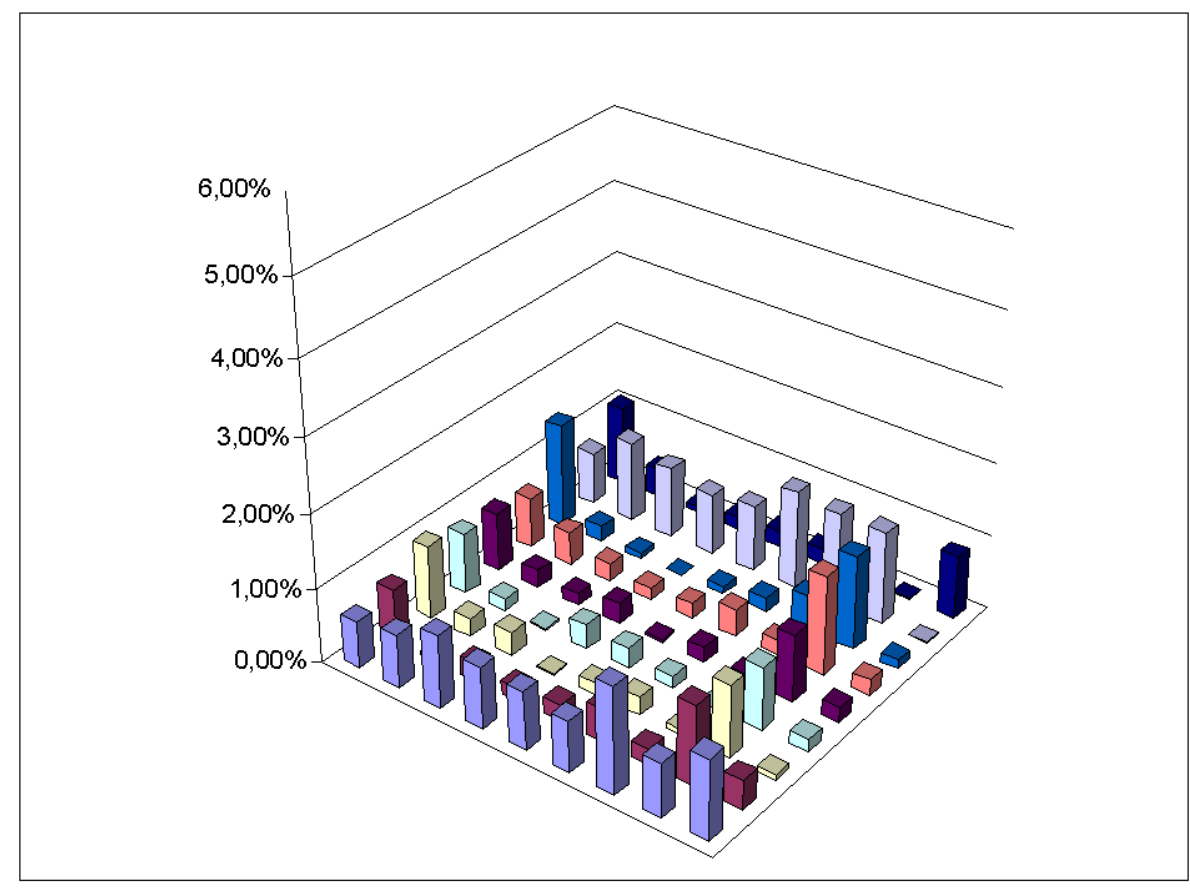

Figure 1: Jäckel regularization

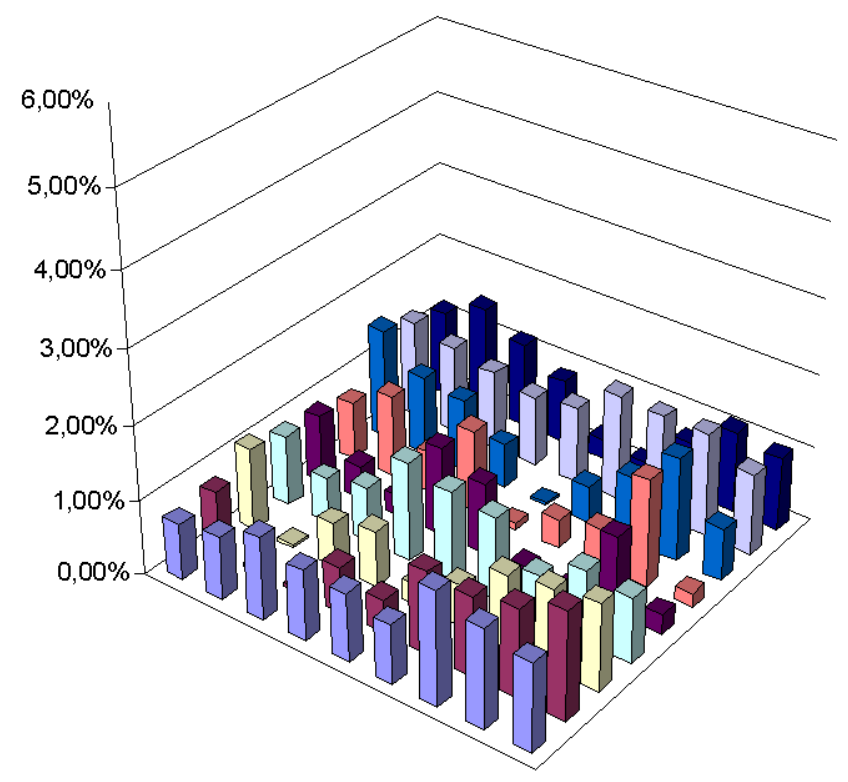

Figure 2: Mishra regularization 


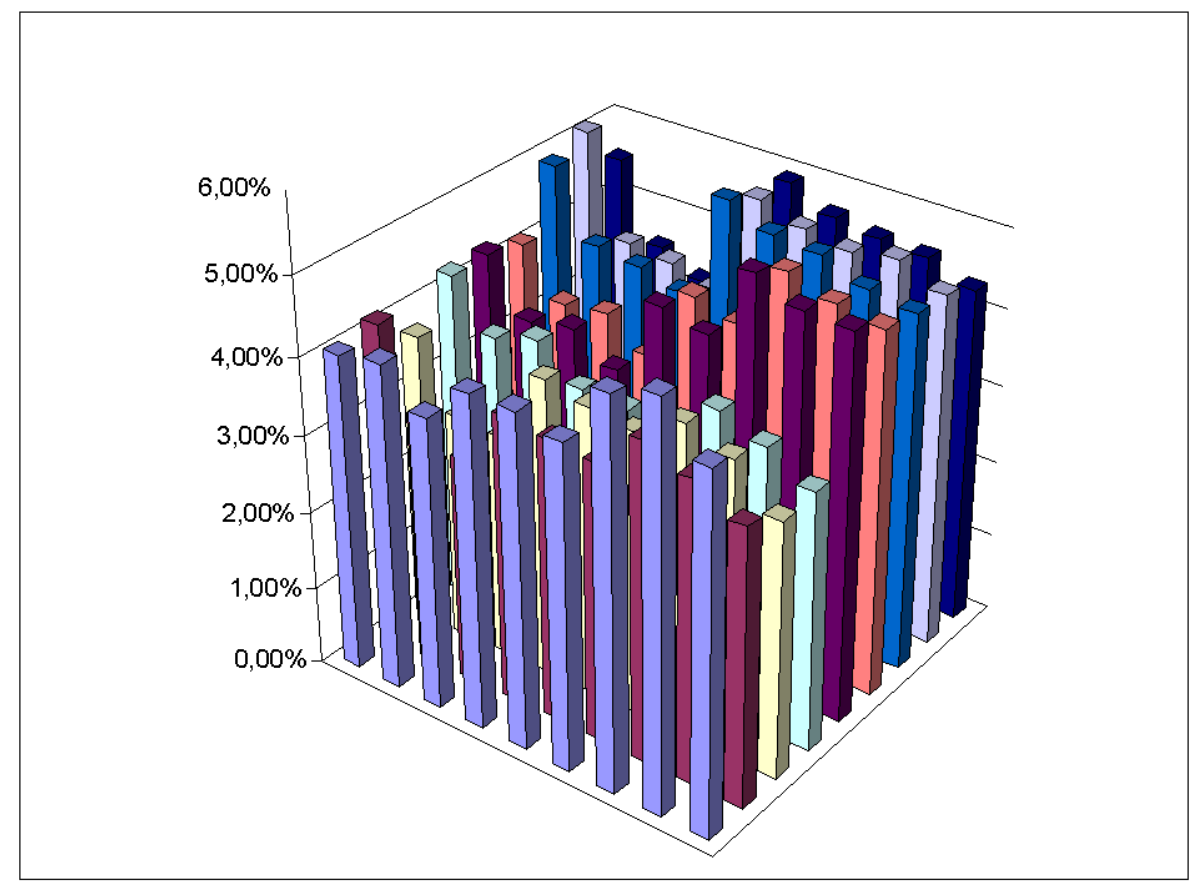

Figure 3: Convex combination regularization

So, in this case the regularization by Jäckel clearly does best.

\section{Calibrating the correlations using historical time series}

As already mentioned, in case we do not have liquid correlations dependent derivatives and we are not provided with the expected cross-asset empirical correlations under the pricing measure, we are left with the only option to determine the correlations historically. We will treat only the transition to the physical measure and the estimation of the correlations $\rho_{i j}$ in the two asset case. The regularization to a valid correlation matrix is identical to the previous section.

\section{$5.1 \quad$ Algorithm}

The input is the $d$-dimensional historical time series $\left(S_{1}\left(t_{k}\right), \ldots, S_{d}\left(t_{k}\right)\right)_{k=0, \ldots, K}$ of the assets in interest. They are then transformed into $\log$ returns $X_{i}\left(t_{k}\right):=\log \frac{X_{i}\left(t_{k}\right)}{X_{i}\left(t_{k-1}\right)}$ and the 
historical empirical correlations $\widehat{\rho}_{i, j: K}^{\mathrm{emp}}$ are defined as in (5).

The next step is to determine the historical mean reversion level of the volatilities of all single-asset models. Using only the historical mean reversion parameters and retaining Heston's original assumption on the shape of the market price of risk, we can deduce the dynamics of the single-asset models under the physical measure.

At the end we calibrate the correlation matrix $\Sigma$ using the historical empirical correlation by the correlation adjustment method presented above. The only difference is that the simulations are being performed under the physical measure rather than the equivalent martingale measure.

\subsection{Dynamics under the physical measure}

According to the original article Heston [1993] the market price of volatility risk has the form $\lambda \cdot \nu$ (we omit the subscripts indicating the assets as we only treat the one dimensional Heston in this section) and consequently the model parameters $\theta$ under the pricing measure and the parameters $\theta^{*}$ under the physical measure are connected via the following relations

$$
\begin{aligned}
& \rho=\rho^{*}, \quad \eta=\eta^{*} \\
& \kappa=\kappa^{*}+\lambda \\
& \bar{\nu}=\frac{\kappa^{*} \bar{\nu}^{*}}{\kappa^{*}+\lambda} .
\end{aligned}
$$

From the above equations we immediately conclude that $\kappa^{*} \cdot \bar{\nu}^{*}=\kappa \cdot \bar{\nu}$ and therefore it is enough to estimate the mean reversion level $\bar{\nu}^{*}$ under the physical measure.

We chose the estimator based on the following well-known convergence.

Proposition 5.1. Let $(S(t), \nu(t))$ be the processes defined in (1) and (2). Let $\pi_{n}=$ $\left(t_{k, n}\right)_{k=1, \ldots, K_{n}(T)}$ be partitions of $[0, T]$, with mesh $\left(\pi_{n}\right)=\max _{i=1, \ldots, K_{n}(T)}\left|t_{i, n}-t_{i-1, n}\right| \rightarrow 0$, for $n \rightarrow \infty$. Then for the $\log$ returns $X\left(t_{k}\right):=\log \frac{S\left(t_{k}\right)}{S\left(t_{k-1}\right)}$

$$
\lim _{T \rightarrow 0} \lim _{n \rightarrow \infty} \frac{1}{T} \sum_{k=1}^{K_{n}(T)} X^{2}\left(t_{k}\right)=\bar{\nu}^{*} \text { almost surely }
$$


Proof. We know that almost surely

$$
\sum_{k=1}^{K_{n}(T)} X^{2}\left(t_{k}\right)=\sum_{k=1}^{K_{n}(T)}\left(\log S\left(t_{k}\right)-\log S\left(t_{k-1}\right)\right)^{2} \underset{n \rightarrow \infty}{\longrightarrow}\langle\log S\rangle_{T}=\int_{0}^{T} \nu(s) \mathrm{d} s
$$

and the ergodic theorem implies

$$
\frac{1}{T} \int_{0}^{T} \nu(s) \mathrm{d} s \underset{T \rightarrow \infty}{\longrightarrow} \bar{\nu} \text { a.s. }
$$

Combining the above facts proves the statement.

A straightforward estimator for the mean reversion level under the physical measure is obtained by stipulating that for large $T$ and every $i=1, \ldots, d$ we have $\sum_{k=1}^{K_{n}(T)} X_{i}^{2}\left(t_{k}\right) \approx$ $T \bar{\nu}_{i}^{*}$, i.e. we set

$$
\widehat{\bar{\nu}}_{i}^{*}:=\frac{1}{T} \sum_{k=1}^{K} X_{i}^{2}\left(t_{k}\right) \text { for all } i=1, \ldots, d
$$

\subsection{Calibration of $\Sigma$}

The two-asset calibration of the correlations $\rho_{i, j}$ can be performed analogously to the procedure in Section 4.1.1, with the technical difference that we do not use the parameters under the risk neutral measure to generate the paths and calculate $\mathbb{E} \widehat{\rho}_{i, j}^{\mathrm{emp}}\left(\rho_{i, j}\right)$, but we rather use the parameters under the physical measure. Obviously the regularization of the matrix obtained by the successive two-asset calibrations in order to end up with a valid correlation matrix does not depend on the measure under which we perform the calibration and is exactly as in Section 4.2.

\section{Conclusion}

In this paper we presented a parsimonious multi-asset Heston model. All single-asset sub-models follow the well-known Heston dynamics and their parameters are calibrated as usual on implied market volatilities. Our focus was on the calibration of the correlation structure between the single-asset marginals in the absence of sufficient liquid cross-asset 
option price data. We presented two general calibration setups corresponding to relevant practical situations: (1) when the empirical cross-asset correlations in the risk neutral world are given by the user and we need to calibrate the correlations between the driving Brownian motions or (2) when they have to be estimated from the historical time series. The theoretical background, including the ergodicity of the multidimensional CIR process, for the proposed estimators are worked out.

The presented model is parsimonious in the sense that $d(d-1) / 2$ asset-asset crosscorrelations are required for a $d$-asset Heston model. This correlation matrix can be used to simulate multi-asset dynamics using the Heston model. We also presented three methods for obtaining a valid correlation matrix, if the empirical matrix given by the user is not or the calibrated cross-asset correlations do not form such a matrix. In addition, we compared the three methods by giving numerical results to state the usefulness of the proposed approaches.

\section{A Ergodicity of the two dimensional CIR process}

Theorem A.1. Let $\nu:=\left(\nu_{1}, \nu_{2}\right)$ be a two dimensional CIR process with parameters $\kappa_{1}, \kappa_{2}, \eta_{1}, \eta_{2}, \bar{\nu}_{1}, \bar{\nu}_{2}>0$ and $\rho \in(-1,1)$, i.e. it is a continuous Markov process with infinitesimal generator

$$
\mathcal{A}=\kappa_{1}\left(\bar{\nu}_{1}-x\right) \partial_{x}+\kappa_{2}\left(\bar{\nu}_{2}-y\right) \partial_{y}+\frac{1}{2} \eta_{1}^{2} x \partial_{x x}+\frac{1}{2} \eta_{2}^{2} y \partial_{y y}+\rho \eta_{1} \eta_{2} \sqrt{x y} \partial_{x y} .
$$

$\nu$ has a stationary probability distribution $\mu_{\infty}(\mathrm{d} x \mathrm{~d} y)$ on $\mathbb{R}_{+}^{2}$, and moreover, for every $\mu$-integrable $f: \mathbb{R}_{+}^{2} \rightarrow \mathbb{R}$ we have for $\mu$-almost all $(x, y)$

$$
\mathbb{P}_{(x, y)}\left[\lim _{T \rightarrow \infty} \frac{1}{T} \int_{0}^{T} f\left(\nu_{1}(t), \nu_{2}(t)\right) \mathrm{d} t=\int f(u, v) \mu(\mathrm{d} u \mathrm{~d} v)\right]=1 .
$$

Remark A.2. We actually need the above result for the function $f(x, y)=\sqrt{x y}$. The fact that $f$ is $\mu$-integrable is a simple consequence of the Hölder inequality. Indeed

$$
\int \sqrt{x y} \mu(\mathrm{d} x \mathrm{~d} y) \leq \sqrt{\int x \mu(\mathrm{d} x \mathrm{~d} y) \int y \mu(\mathrm{d} x \mathrm{~d} y)}=\sqrt{\bar{\nu}_{1} \cdot \bar{\nu}_{2}} .
$$


Proof. The statement of the theorem is a special case of Theorem 5.1, Chapter IV of Has'minskii. However, we need verify that the following condition holds: there is a bounded domain $D$ in $\mathbb{R}_{+}^{2}$ having the following properties:

(1) The smallest eigenvalue of the diffusion matrix of the generator $\mathcal{A}$ is bounded away from zero on $D$.

(2) For every $(x, y) \in \mathbb{R}_{+}^{2} \backslash D$ the mean time $\tau$ at which a trajectory $\left(\nu_{1}^{x}(t), \nu_{2}^{y}(t)\right)$ started at $(x, y)$ reaches $U$ is finite, and even $\sup _{(x, y) \in K} \tau<\infty$ for every compact set $K \subset \mathbb{R}_{+}^{2} \backslash D$.

We first present a rather concise solution in the case where both one dimensional CIR processes do not reach the origin (i.e. the stability condition $2 \kappa_{i} \bar{\nu}_{i} \geq \eta_{i}^{2}$ is satisfied). In this case the state space is $E:=(0, \infty)^{2}$ as $P\left(\nu_{i}(t)>0 \forall t\right)=1, i=1,2$.

The first condition will obviously be satisfied if the closure of $D$ is contained in $E$. Indeed, the eigenvalues are given by

$$
\lambda_{1,2}=\frac{\bar{\eta}_{1}^{2} x+\bar{\eta}_{2}^{2} y \pm \sqrt{\left(\bar{\eta}_{1}^{2} x+\bar{\eta}_{2}^{2} y\right)^{2}+4\left(\rho^{2}-1\right) \bar{\eta}_{1}^{2} \bar{\eta}_{2}^{2} x y}}{2},
$$

which can be uniformly bounded away from zero on any compact set contained in $E$ as long as $\rho^{2}<1$.

The second condition per definition actually states that the process $\eta$ has to be $D$ recurrent (see Chapter III, Section 7 in Has'minskii [1980]). According to Theorem 7.1 in Chapter III in the same source, a sufficient condition for the $D$-recurrence is the existence of a nonnegative (Lyapunov) function $V(s, x)$ on $R_{+} \times D^{C}$, twice continuously differentiable in the space variable and continuously differentiable in the time variable and such that

$$
\mathcal{A} V \leq-\delta \text { for some positive } \delta>0 \text { on } R_{+} \times D^{C} .
$$

As $\nu$ is a time homogeneous diffusion we consider Lyapunov functions depending only on the state variable. We take

$$
V(x, y)=a x \ln x+b y \ln y+c: E \rightarrow \mathbb{R}
$$


where $a, b, c>0 . V$ is a $C^{2}$ function on $E$. Since the mapping $x \mapsto x \ln x$ is bounded from below on $E$ for every set of parameters $a, b>0$ we can achieve $V(x, y)>0$ on $E$ by choosing $c$ big enough. Now observe that

$$
\begin{aligned}
\mathcal{A} V(x, y)= & \kappa_{1}\left(\bar{\nu}_{1}-x\right) a(1+\ln x)+\kappa_{2}\left(\bar{\nu}_{2}-y\right) b(1+\ln y)+\frac{1}{2}\left(\eta_{1}^{2} a+\eta_{2}^{2} b\right) \\
= & -\kappa_{1} a(x+x \ln x)+\kappa_{1} a \bar{\nu}_{1} \ln x-\kappa_{2} b(y+y \ln y)+\kappa_{2} b \bar{\nu}_{2} \ln y \\
& +\frac{1}{2}\left(\eta_{1}^{2} a+\eta_{2}^{2} b\right)+\kappa_{1} a \bar{\nu}_{1}+\kappa_{2} b \bar{\nu}_{2} .
\end{aligned}
$$

Since of course $x \ln x$ and $y \ln y$ outperform the other terms for large $x$ and $y$, the set $M$ on which $\mathcal{A} V$ is bigger than say -1 is a bounded subset of $E$. Furthermore, letting $x \rightarrow 0$ we have $\mathcal{A} V(x, y) \rightarrow-\infty$ for all $y>0$ and analogously for $y \rightarrow 0$ we get $\mathcal{A} V(x, y) \rightarrow-\infty$ for arbitrary $x>0$. Thus we conclude that the closure of $M$ is still contained in $E$. Indeed, assume e.g. $\left(x_{0}, 0\right)$ is in the closure of $M$. Then we would have a sequence $M \ni\left(x_{n}, y_{n}\right) \rightarrow\left(x_{0}, 0\right)$ and we would get $-\infty=\lim _{n \rightarrow \infty} \mathcal{A} V\left(x_{n}, y_{n}\right) \geq-1$.

Summarizing, $M$ is a bounded subset of $E$, such that the closure of $M$ is also in $E$ and we have that $\mathcal{A} V \leq-1$ on $M^{C}$. The nonnegativity of $V$ on the whole $E$ is achieved by setting $c>0$ big enough. This verifies the conditions (1) and (2) stated in the beginning of the proof.

We will now sketch the proof in the general case. We need to set $E=[0, \infty)^{2}$ as the one dimensional CIR process may reach the zero boundary. For small enough $\epsilon>0$ define function $g^{\epsilon}:[0, \infty)$ to be the quadratic truncation of $x \ln x$, i.e.

$$
g(x)=\left\{\begin{array}{cc}
x \ln x & \text { if } x>\epsilon \\
a_{\epsilon} x^{2}+b_{\epsilon} x+c_{\epsilon} & x \in[0, \epsilon]
\end{array},\right.
$$

where the coefficients $a_{\epsilon}, b_{\epsilon}$ and $c_{\epsilon}$ are chosen in such a manner that the function $g(x)$ is $C^{2}$. We now consider the Lyapunov function

$$
V^{\epsilon}(x, y)=a g^{\epsilon}(x)+b g^{\epsilon}(y)+c: E \rightarrow \mathbb{R}
$$

with $a, b, c>0$. The nonnegativity can be achieved as above by choosing $c$ large enough. An analogous leading term argument implies that the set $M$ on which $\mathcal{A} V^{\epsilon}(x, y)$ is bigger than -1 is bounded. A rather technical calculation shows that by choosing $\epsilon$ small enough 
we can make $\mathcal{A} V^{\epsilon}(x, y) \leq-2$ on the boundary of $E$. Clearly, there is no surprise as we approximate the case, which we have worked out above. This, however, proves that actually the closure of $M$ is contained in the interior of $E$, as otherwise we would have for example a sequence $M \ni\left(x_{n}, y_{n}\right) \rightarrow\left(x_{0}, 0\right)$ and we would get $-2 \geq \lim _{n \rightarrow \infty} \mathcal{A} V^{\epsilon}\left(x_{n}, y_{n}\right) \geq-1$.

Therefore the set $M$ satisfies the requirements of the conditions (1) and (2) in the beginning of the proof and we are done.

An alternative way would be the case when $g^{\epsilon}$ is the linear rather than quadratic truncation of $x \ln x$. This would actually also formally suffice even if the resulting $V^{\epsilon}$ is only a $C^{1}$ rather than $C^{2}$ function. The reason that it still works is that the proof of Theorem 8.1 in Chapter III in Has'minskii which we invoke relies on Itô's formula and it still holds for $C^{1}$ functions with existing and continuous second derivatives everywhere but on a countable exception set and having bounded second derivatives around the singularities.

\section{References}

L.B.G. Andersen. Efficient simulation of the heston stochastic volatility model. http: //papers.ssrn.com/sol3/papers.cfm?abstract_id=946405, 2007.

C. Gourieroux. Continuous time Wishart process for stochastic risk. Econometric Reviews, 25(2):177-217, 2006.

R.Z. Has'minskii. Stochastic stability of differential equations, vol. 7 of Monographs and Textbooks on Mechanics of Solids and Fluids: Mechanics and Analysis. Sijthoff \& Noordhoff, Alphen aan den Rijn, 1980.

S.L. Heston. A closed-form solution for options with stochastic volatility with applications to bond and currency options. Review of financial studies, pages 327-343, 1993.

P. Jäckel. Monte Carlo methods in finance. John Wiley \& Sons, Ltd. West Sussex, 2002. 
R. Lord, R. Koekkoek, and D.J.C. Van Dijk. A comparison of biased simulation schemes for stochastic volatility models. Journal of Quantitative Finance, 2008.

S.K. Mishra. Optimal solution of the nearest correlation matrix problem by minimization of the maximum norm. http://mpra.ub.uni-muenchen.de/1783/, 2004. 


\section{Published reports of the Fraunhofer ITWM}

The PDF-files of the following reports are available under:

\section{www.itwm.fraunhofer.de/de/ zentral_berichte/berichte}

1. D. Hietel, K. Steiner, J. Struckmeier A Finite - Volume Particle Method for Compressible Flows (19 pages, 1998)

2. M. Feldmann, S. Seibold

Damage Diagnosis of Rotors: Application of Hilbert Transform and Multi-Hypothesis Testing

Keywords: Hilbert transform, damage diagnosis,

Kalman filtering, non-linear dynamics

(23 pages, 1998)

3. Y. Ben-Haim, S. Seibold

Robust Reliability of Diagnostic MultiHypothesis Algorithms: Application to Rotating Machinery

Keywords: Robust reliability, convex models, Kalman filtering, multi-hypothesis diagnosis, rotating machinery, crack diagnosis

(24 pages, 1998)

\section{F.-Th. Lentes, N. Siedow}

Three-dimensional Radiative Heat Transfer in Glass Cooling Processes

(23 pages, 1998)

5. A. Klar, R. Wegener

A hierarchy of models for multilane vehicular traffic

Part I: Modeling

(23 pages, 1998)

Part II: Numerical and stochastic investigations (17 pages, 1998)

\section{A. Klar, N. Siedow}

Boundary Layers and Domain Decomposition for Radiative Heat Transfer and Diffusion Equations: Applications to Glass Manufacturing Processes

(24 pages, 1998)

7. I. Choquet

Heterogeneous catalysis modelling and numerical simulation in rarified gas flows Part l: Coverage locally at equilibrium (24 pages, 1998)

8. J. Ohser, B. Steinbach, C. Lang Efficient Texture Analysis of Binary Images (17 pages, 1998)

9. J. Orlik

Homogenization for viscoelasticity of the integral type with aging and shrinkage (20 pages, 1998)

10. J. Mohring

Helmholtz Resonators with Large Aperture (21 pages, 1998)
11. H. W. Hamacher, A. Schöbel On Center Cycles in Grid Graphs (15 pages, 1998)

12. H. W. Hamacher, K.-H. Küfer Inverse radiation therapy planning a multiple objective optimisation approach (14 pages, 1999)

13. C. Lang, J. Ohser, R. Hilfer On the Analysis of Spatial Binary Images (20 pages, 1999)

14. M. Junk

On the Construction of Discrete Equilibrium Distributions for Kinetic Schemes (24 pages, 1999)

15. M. Junk, S. V. Raghurame Rao

A new discrete velocity method for NavierStokes equations

(20 pages, 1999)

16. H. Neunzert

Mathematics as a Key to Key Technologies (39 pages (4 PDF-Files), 1999)

17. J. Ohser, K. Sandau

Considerations about the Estimation of the Size Distribution in Wicksell's Corpuscle Problem

(18 pages, 1999)

18. E. Carrizosa, H. W. Hamacher, R. Klein, S. Nickel

Solving nonconvex planar location problems by finite dominating sets

Keywords: Continuous Location, Polyhedral Gauges, Finite Dominating Sets, Approximation, Sandwich Algorithm, Greedy Algorithm

(19 pages, 2000)

\section{A. Becker}

A Review on Image Distortion Measures Keywords: Distortion measure, human visual system (26 pages, 2000)

20. H. W. Hamacher, M. Labbé, S. Nickel, T. Sonneborn

Polyhedral Properties of the Uncapacitated Multiple Allocation Hub Location Problem Keywords: integer programming, hub location, facility location, valid inequalities, facets, branch and cut (21 pages, 2000)

21. H. W. Hamacher, A. Schöbel

Design of Zone Tariff Systems in Public Transportation

(30 pages, 2001)

22. D. Hietel, M. Junk, R. Keck, D. Teleaga The Finite-Volume-Particle Method for Conservation Laws (16 pages, 2001)

23. T. Bender, H. Hennes, J. Kalcsics, M. T. Melo, S. Nickel

Location Software and Interface with GIS and Supply Chain Management Keywords: facility location, software development, geographical information systems, supply chain management

(48 pages, 2001)
24. H. W. Hamacher, S. A. Tjandra Mathematical Modelling of Evacuation Problems: A State of Art (44 pages, 2001)

25. J. Kuhnert, S. Tiwari

Grid free method for solving the Poisson equation

Keywords: Poisson equation, Least squares method, Grid free method

(19 pages, 2001)

26. T. Götz, H. Rave, D. Reinel-Bitzer, K. Steiner, $\mathrm{H}$. Tiemeier

Simulation of the fiber spinning process Keywords: Melt spinning, fiber model, Lattice Boltzmann, CFD

(19 pages, 2001)

\section{A. Zemitis}

On interaction of a liquid film with an obstacle Keywords: impinging jets, liquid film, models, numerical solution, shape

22 pages, 2001)

\section{I. Ginzburg, K. Steiner}

Free surface lattice-Boltzmann method to model the filling of expanding cavities by Bingham Fluids

Keywords: Generalized LBE, free-surface phenomena interface boundary conditions, filling processes, Bing ham viscoplastic model, regularized models

(22 pages, 2001)

\section{H. Neunzert}

"Denn nichts ist für den Menschen als Menschen etwas wert, was er nicht mit Leidenschaft tun kann

Vortrag anlässlich der Verleihung des Akademiepreises des Landes RheinlandPfalz am 21.11.2001

Keywords: Lehre, Forschung, angewandte Mathematik, Mehrskalenanalyse, Strömungsmechanik (18 pages, 2001)

30. J. Kuhnert, S. Tiwari

Finite pointset method based on the projection method for simulations of the incompressible Navier-Stokes equations

Keywords: Incompressible Navier-Stokes equations, Meshfree method, Projection method, Particle scheme, Least squares approximation

AMS subject classification: 76D05, 76M28

(25 pages, 2001)

31. R. Korn, M. Krekel

Optimal Portfolios with Fixed Consumption or Income Streams

Keywords: Portfolio optimisation, stochastic control, HJB equation, discretisation of control problems (23 pages, 2002)

32. M. Krekel

Optimal portfolios with a loan dependent credit spread

Keywords: Portfolio optimisation, stochastic control, HJB equation, credit spread, log utility, power utility, non-linear wealth dynamics

(25 pages, 2002)

33. J. Ohser, W. Nagel, K. Schladitz

The Euler number of discretized sets - on the choice of adjacency in homogeneous lattices Keywords: image analysis, Euler number, neighborhod relationships, cuboidal lattice

(32 pages, 2002) 
34. I. Ginzburg, K. Steiner

Lattice Boltzmann Model for Free-Surface flow and Its Application to Filling Process in Casting

Keywords: Lattice Boltzmann models; free-surface phenomena; interface boundary conditions; filling processes; injection molding; volume of fluid method; interface boundary conditions; advection-schemes; upwind-schemes

(54 pages, 2002)

35. M. Günther, A. Klar, T. Materne, R. Wegener

Multivalued fundamental diagrams and stop and go waves for continuum traffic equations Keywords: traffic flow, macroscopic equations, kinetic derivation, multivalued fundamental diagram, stop and go waves, phase transitions

(25 pages, 2002)

36. S. Feldmann, P. Lang, D. Prätzel-Wolters Parameter influence on the zeros of network determinants

Keywords: Networks, Equicofactor matrix polynomials, Realization theory, Matrix perturbation theory

(30 pages, 2002)

\section{K. Koch, J. Ohser, K. Schladitz} Spectral theory for random closed sets and estimating the covariance via frequency space

Keywords: Random set, Bartlett spectrum, fast Fourier transform, power spectrum

(28 pages, 2002)

\section{D. d'Humières, I. Ginzburg}

Multi-reflection boundary conditions for lattice Boltzmann models

Keywords: lattice Boltzmann equation, boudary condistions, bounce-back rule, Navier-Stokes equation

(72 pages, 2002)

\section{R. Korn}

\section{Elementare Finanzmathematik}

Keywords: Finanzmathematik, Aktien, Optionen, Portfolio-Optimierung, Börse, Lehrerweiterbildung, Mathematikunterricht

(98 pages, 2002)

40. J. Kallrath, M. C. Müller, S. Nickel

Batch Presorting Problems:

Models and Complexity Results

Keywords: Complexity theory, Integer programming,

Assigment, Logistics

(19 pages, 2002)

\section{J. Linn}

On the frame-invariant description of the phase space of the Folgar-Tucker equation Key words: fiber orientation, Folgar-Tucker equation, injection molding

(5 pages, 2003)

\section{T. Hanne, S. Nickel}

A Multi-Objective Evolutionary Algorithm for Scheduling and Inspection Planning in Software Development Projects

Key words: multiple objective programming, project management and scheduling, software development, evolutionary algorithms, efficient set

(29 pages, 2003)

43. T. Bortfeld, K.-H. Küfer, M. Monz,

A. Scherrer, C. Thieke, H. Trinkaus

Intensity-Modulated Radiotherapy - A Large Scale Multi-Criteria Programming Problem
Keywords: multiple criteria optimization, representative systems of Pareto solutions, adaptive triangulation, clustering and disaggregation techniques, visualization of Pareto solutions, medical physics, external beam radiotherapy planning, intensity modulated radiotherapy (31 pages, 2003)

\section{T. Halfmann, T. Wichmann}

Overview of Symbolic Methods in Industrial Analog Circuit Design

Keywords: $C A D$, automated analog circuit design, sym bolic analysis, computer algebra, behavioral modeling, system simulation, circuit sizing, macro modeling, differential-algebraic equations, index

(17 pages, 2003)

\section{S. E. Mikhailov, J. Orlik}

Asymptotic Homogenisation in Strength and Fatigue Durability Analysis of Composites

Keywords: multiscale structures, asymptotic homogenization, strength, fatigue, singularity, non-local conditions

(14 pages, 2003)

46. P. Domínguez-Marín, P. Hansen, N. Mladenovi'c , S. Nickel

Heuristic Procedures for Solving the Discrete Ordered Median Problem

Keywords: genetic algorithms, variable neighborhood search, discrete facility location

(31 pages, 2003)

47. N. Boland, P. Domínguez-Marín, S. Nickel, J. Puerto

Exact Procedures for Solving the Discrete Ordered Median Problem

Keywords: discrete location, Integer programming

(41 pages, 2003)

48. S. Feldmann, P. Lang

Padé-like reduction of stable discrete linear systems preserving their stability Keywords: Discrete linear systems, model reduction, stability, Hankel matrix, Stein equation

(16 pages, 2003)

49. J. Kallrath, S. Nickel

A Polynomial Case of the Batch Presorting Problem

Keywords: batch presorting problem, online optimization, competetive analysis, polynomial algorithms, logistics (17 pages, 2003)

\section{T. Hanne, H. L. Trinkaus}

knowCube for MCDM -

Visual and Interactive Support for Multicriteria Decision Making

Key words: Multicriteria decision making, knowledge management, decision support systems, visual interfaces, interactive navigation, real-life applications. (26 pages, 2003)

51. O. lliev, V. Laptev

On Numerical Simulation of Flow Through Oil Filters

Keywords: oil filters, coupled flow in plain and porous media, Navier-Stokes, Brinkman, numerical simulation (8 pages, 2003)

52. W. Dörfler, O. Iliev, D. Stoyanov, D. Vassileva On a Multigrid Adaptive Refinement Solver for Saturated Non-Newtonian Flow in Porous Media

Keywords: Nonlinear multigrid, adaptive refinement, non-Newtonian flow in porous media

(17 pages, 2003)
53. S. Kruse

On the Pricing of Forward Starting Options under Stochastic Volatility

Keywords: Option pricing, forward starting options, Heston model, stochastic volatility, cliquet options (11 pages, 2003)

54. O. Iliev, D. Stoyanov

Multigrid - adaptive local refinement solver for incompressible flows

Keywords: Navier-Stokes equations, incompressible flow projection-type splitting, SIMPLE, multigrid methods, adaptive local refinement, lid-driven flow in a cavity (37 pages, 2003)

\section{V. Starikovicius}

The multiphase flow and heat transfer in porous media

Keywords: Two-phase flow in porous media, various formulations, global pressure, multiphase mixture model, numerical simulation

(30 pages, 2003)

\section{P. Lang, A. Sarishvili, A. Wirsen}

Blocked neural networks for knowledge extraction in the software development process Keywords: Blocked Neural Networks, Nonlinear Regression, Knowledge Extraction, Code Inspection (21 pages, 2003)

\section{H. Knaf, P. Lang, S. Zeiser}

Diagnosis aiding in Regulation

Thermography using Fuzzy Logic

Keywords: fuzzy logic, knowledge representation, expert system

(22 pages, 2003)

58. M. T. Melo, S. Nickel, F. Saldanha da Gama Largescale models for dynamic multicommodity capacitated facility location Keywords: supply chain management, strategic planning, dynamic location, modeling (40 pages, 2003)

\section{J. Orlik}

Homogenization for contact problems with periodically rough surfaces

Keywords: asymptotic homogenization, contact problems (28 pages, 2004)

60. A. Scherrer, K.-H. Küfer, M. Monz,

F. Alonso, T. Bortfeld

IMRT planning on adaptive volume structures - a significant advance of computational complexity

Keywords: Intensity-modulated radiation therapy (IMRT), inverse treatment planning, adaptive volume structures, hierarchical clustering, local refinement, adaptive clustering, convex programming, mesh generation, multi-grid methods

(24 pages, 2004)

\section{D. Kehrwald}

Parallel lattice Boltzmann simulation of complex flows

Keywords: Lattice Boltzmann methods, parallel computing, microstructure simulation, virtual material design, pseudo-plastic fluids, liquid composite moulding (12 pages, 2004)

62. O. Iliev, J. Linn, M. Moog, D. Niedziela, V. Starikovicius

On the Performance of Certain Iterative Solvers for Coupled Systems Arising in Discretization of Non-Newtonian Flow Equations 
Keywords: Performance of iterative solvers, Preconditioners, Non-Newtonian flow

(17 pages, 2004)

\section{R. Ciegis, O. Iliev, S. Rief, K. Steiner}

On Modelling and Simulation of Different Regimes for Liquid Polymer Moulding Keywords: Liquid Polymer Moulding, Modelling, Simulation, Infiltration, Front Propagation, non-Newtonian flow in porous media

(43 pages, 2004)

\section{T. Hanne, H. Neu}

Simulating Human Resources in

\section{Software Development Processes}

Keywords: Human resource modeling, software process, productivity, human factors, learning curve

(14 pages, 2004)

\section{O. Iliev, A. Mikelic, P. Popov}

Fluid structure interaction problems in de-

formable porous media: Toward permeability of deformable porous media

Keywords: fluid-structure interaction, deformable porous media, upscaling, linear elasticity, stokes, finite elements

(28 pages, 2004)

66. F. Gaspar, O. Iliev, F. Lisbona, A. Naumovich, P. Vabishchevich

On numerical solution of 1-D poroelasticity equations in a multilayered domain Keywords: poroelasticity, multilayered material, finite volume discretization, MAC type grid

(41 pages, 2004)

67. J. Ohser, K. Schladitz, K. Koch, M. Nöthe Diffraction by image processing and its application in materials science

Keywords: porous microstructure, image analysis, random set, fast Fourier transform, power spectrum, Bartlett spectrum

(13 pages, 2004)

\section{H. Neunzert}

\section{Mathematics as a Technology: Challenges} for the next 10 Years

Keywords: applied mathematics, technology, modelling, simulation, visualization, optimization, glass processing, spinning processes, fiber-fluid interaction, trubulence effects, topological optimization, multicriteria optimiza tion, Uncertainty and Risk, financial mathematics, Malliavin calculus, Monte-Carlo methods, virtual material design, filtration, bio-informatics, system biology

(29 pages, 2004)

69. R. Ewing, O. lliev, R. Lazarov, A. Naumovich On convergence of certain finite difference discretizations for 1D poroelasticity interface problems

Keywords: poroelasticity, multilayered material, finite volume discretizations, MAC type grid, error estimates (26 pages, 2004 )

70. W. Dörfler, O. Iliev, D. Stoyanov, D. Vassileva On Efficient Simulation of Non-Newtonian Flow in Saturated Porous Media with a Multigrid Adaptive Refinement Solver Keywords: Nonlinear multigrid, adaptive renement non-Newtonian in porous media (25 pages, 2004)

\section{J. Kalcsics, S. Nickel, M. Schröde}

Towards a Unified Territory Design Approach - Applications, Algorithms and GIS Integration Keywords: territory desgin, political districting, sales territory alignment, optimization algorithms, Geographical Information Systems

(40 pages, 2005)
72. K. Schladitz, S Peters, D Reinel-Bitzer, A. Wiegmann, J. Ohser

Design of acoustic trim based on geometric modeling and flow simulation for non-woven Keywords: random system of fibers, Poisson line process, flow resistivity, acoustic absorption, Lattice-Boltzmann method, non-woven (21 pages, 2005)

\section{V. Rutka, A. Wiegmann}

Explicit Jump Immersed Interface Method for virtual material design of the effective elastic moduli of composite materials Keywords: virtual material design, explicit jump immersed interface method, effective elastic moduli, composite materials

(22 pages, 2005)

\section{T. Hanne}

Eine Übersicht zum Scheduling von Baustellen Keywords: Projektplanung, Scheduling, Bauplanung, Bauindustrie

(32 pages, 2005)

75. J. Linn

The Folgar-Tucker Model as a Differetial Algebraic System for Fiber Orientation Calculation

Keywords: fiber orientation, Folgar-Tucker model, invariants, algebraic constraints, phase space, trace stability

(15 pages, 2005)

76. M. Speckert, K. Dreßler, H. Mauch, A. Lion, G. J. Wierda

Simulation eines neuartigen Prüfsystems für Achserprobungen durch MKS-Modellierung einschließlich Regelung Keywords: virtual test rig, suspension testing, multibody simulation, modeling hexapod test rig, opti mization of test rig configuration

(20 pages, 2005)

77. K.-H. Küfer, M. Monz, A. Scherrer, P. Süss, F. Alonso, A. S. A. Sultan, Th. Bortfeld,

D. Craft, Chr. Thieke

Multicriteria optimization in intensity modulated radiotherapy planning Keywords: multicriteria optimization, extreme solutions, real-time decision making, adaptive approxima tion schemes, clustering methods, IMRT planning, reverse engineering

(51 pages, 2005)

78. S. Amstutz, H. Andrä

A new algorithm for topology optimization using a level-set method

Keywords: shape optimization, topology optimization topological sensitivity, level-set

(22 pages, 2005)

\section{N. Ettrich}

Generation of surface elevation models for urban drainage simulation

Keywords: Flooding, simulation, urban elevation models, laser scanning

(22 pages, 2005

80. H. Andrä, J. Linn, I. Matei, I. Shklyar, K. Steiner, E. Teichmann

OPTCAST - Entwicklung adäquater Struk turoptimierungsverfahren für Gießereien Technischer Bericht (KURZFASSUNG) Keywords: Topologieoptimierung, Level-Set-Methode Gießprozesssimulation, Gießtechnische Restriktionen, CAE-Kette zur Strukturoptimierung

(77 pages, 2005)
81. N. Marheineke, R. Wegener Fiber Dynamics in Turbulent Flows Part I: General Modeling Framework Keywords: fiber-fluid interaction; Cosserat rod; turbuence modeling: Kolmogorov's energy spectrum; double-velocity correlations; differentiable Gaussian fields (20 pages, 2005)

\section{Part II: Specific Taylor Drag}

Keywords: flexible fibers; $k-\varepsilon$ turbulence model; fiber-turbulence interaction scales; air drag; random Gaussian aerodynamic force; white noise; stochastic differential equations; ARMA process

(18 pages, 2005)

\section{C. H. Lampert, O. Wirjadi}

An Optimal Non-Orthogonal Separation of the Anisotropic Gaussian Convolution Filter Keywords: Anisotropic Gaussian filter, linear filtering, or entation space, $n D$ image processing, separable filters (25 pages, 2005)

83. H. Andrä, D. Stoyanov

Error indicators in the parallel finite element solver for linear elasticity DDFEM Keywords: linear elasticity, finite element method, hierarchical shape functions, domain decom-position, parallel implementation, a posteriori error estimates (21 pages, 2006)

84. M. Schröder, I. Solchenbach Optimization of Transfer Quality in Regional Public Transit

Keywords: public transit, transfer quality, quadratic assignment problem

(16 pages, 2006

85. A. Naumovich, F. J. Gaspar

On a multigrid solver for the three-dimensional Biot poroelasticity system in multilayered domains

Keywords: poroelasticity, interface problem, multigrid, operator-dependent prolongation

(11 pages, 2006)

86. S. Panda, R. Wegener, N. Marheineke Slender Body Theory for the Dynamics of Curved Viscous Fibers

Keywords: curved viscous fibers; fluid dynamics; NavierStokes equations; free boundary value problem; asymptotic expansions; slender body theory

(14 pages, 2006)

87. E. Ivanov, H. Andrä, A. Kudryavtsev Domain Decomposition Approach for Automatic Parallel Generation of Tetrahedral Grids Key words: Grid Generation, Unstructured Grid, Delau nay Triangulation, Parallel Programming, Domain Decomposition, Load Balancing

(18 pages, 2006)

88. S. Tiwari, S. Antonov, D. Hietel, J. Kuhnert, R. Wegener

A Meshfree Method for Simulations of In teractions between Fluids and Flexible Structures

Key words: Meshfree Method, FPM, Fluid Structure Interaction, Sheet of Paper, Dynamical Coupling (16 pages, 2006)

89. R. Ciegis, O. Iliev, V. Starikovicius, K. Steine Numerical Algorithms for Solving Problems of Multiphase Flows in Porous Media Keywords: nonlinear algorithms, finite-volume method software tools, porous media, flows

16 pages, 2006) 
90. D. Niedziela, O. Iliev, A. Latz

On 3D Numerical Simulations of Viscoelastic Fluids

Keywords: non-Newtonian fluids, anisotropic viscosity, integral constitutive equation

(18 pages, 2006)

\section{A. Winterfeld}

Application of general semi-infinite Programming to Lapidary Cutting Problems Keywords: large scale optimization, nonlinear programming, general semi-infinite optimization, design centering, clustering

(26 pages, 2006)

\section{J. Orlik, A. Ostrovska}

Space-Time Finite Element Approximation and Numerical Solution of Hereditary Linear Viscoelasticity Problems

Keywords: hereditary viscoelasticity; kern approximation by interpolation; space-time finite element approximation, stability and a priori estimate

(24 pages, 2006)

93. V. Rutka, A. Wiegmann, H. Andrä EJIIM for Calculation of effective Elastic Moduli in 3D Linear Elasticity

Keywords: Elliptic PDE, linear elasticity, irregular domain, finite differences, fast solvers, effective elastic moduli

(24 pages, 2006)

\section{A. Wiegmann, A. Zemitis}

EJ-HEAT: A Fast Explicit Jump Harmonic Averaging Solver for the Effective Heat Conductivity of Composite Materials Keywords: Stationary heat equation, effective thermal conductivity, explicit jump, discontinuous coefficients, virtual material design, microstructure simulation, EJ-HEAT

(21 pages, 2006)

\section{A. Naumovich}

On a finite volume discretization of the three-dimensional Biot poroelasticity system in multilayered domains

Keywords: Biot poroelasticity system, interface problems, finite volume discretization, finite difference method (21 pages, 2006)

\section{M. Krekel, J. Wenzel}

A unified approach to Credit Default Swaption and Constant Maturity Credit Default Swap valuation

Keywords: LIBOR market model, credit risk, Credit Default Swaption, Constant Maturity Credit Default Swapmethod

(43 pages, 2006)

\section{A. Dreyer}

\section{Interval Methods for Analog Circiuts}

Keywords: interval arithmetic, analog circuits, tolerance analysis, parametric linear systems, frequency response, symbolic analysis, CAD, computer algebra

(36 pages, 2006)

98. N. Weigel, S. Weihe, G. Bitsch, K. Dreßler Usage of Simulation for Design and Optimization of Testing

Keywords: Vehicle test rigs, MBS, control, hydraulics,

testing philosophy

(14 pages, 2006)

99. H. Lang, G. Bitsch, K. Dreßler, M. Speckert Comparison of the solutions of the elastic and elastoplastic boundary value problems
Keywords: Elastic BVP, elastoplastic BVP, variational inequalities, rate-independency, hysteresis, linear kinematic hardening, stop- and play-operator

(21 pages, 2006)

100. M. Speckert, K. Dreßler, H. Mauch MBS Simulation of a hexapod based suspension test rig

Keywords: Test rig, MBS simulation, suspension,

hydraulics, controlling, design optimization

(12 pages, 2006)

101. S. Azizi Sultan, K.-H. Küfer

A dynamic algorithm for beam orientations in multicriteria IMRT planning

Keywords: radiotherapy planning, beam orientation optimization, dynamic approach, evolutionary algorithm, global optimization

(14 pages, 2006)

102. T. Götz, A. Klar, N. Marheineke, R. Wegener A Stochastic Model for the Fiber Lay-down Process in the Nonwoven Production Keywords: fiber dynamics, stochastic Hamiltonian system, stochastic averaging

(17 pages, 2006)

103. Ph. Süss, K.-H. Küfer

Balancing control and simplicity: a variable aggregation method in intensity modulated radiation therapy planning

Keywords: IMRT planning, variable aggregation, clustering methods

(22 pages, 2006)

104. A. Beaudry, G. Laporte, T. Melo, S. Nickel Dynamic transportation of patients in hospitals

Keywords: in-house hospital transportation, dial-a-ride, dynamic mode, tabu search

(37 pages, 2006)

105. Th. Hanne

Applying multiobjective evolutionary algorithms in industrial projects

Keywords: multiobjective evolutionary algorithms, discrete optimization, continuous optimization, electronic circuit design, semi-infinite programming, scheduling (18 pages, 2006)

106. J. Franke, S. Halim

Wild bootstrap tests for comparing signals and images

Keywords: wild bootstrap test, texture classification, textile quality control, defect detection, kernel estimate, nonparametric regression

(13 pages, 2007)

107. Z. Drezner, S. Nickel

Solving the ordered one-median problem in the plane

Keywords: planar location, global optimization, ordered median, big triangle small triangle method, bounds, numerical experiments

(21 pages, 2007)

108. Th. Götz, A. Klar, A. Unterreiter, R. Wegener

Numerical evidance for the non-existing of solutions of the equations desribing rotational fiber spinning

Keywords: rotational fiber spinning, viscous fibers, boundary value problem, existence of solutions (11 pages, 2007)
109. Ph. Süss, K.-H. Küfer

Smooth intensity maps and the BortfeldBoyer sequencer

Keywords: probabilistic analysis, intensity modulated radiotherapy treatment (IMRT), IMRT plan application step-and-shoot sequencing

(8 pages, 2007)

110. E. Ivanov, O. Gluchshenko, H. Andrä, A. Kudryavtsev

Parallel software tool for decomposing and meshing of $3 d$ structures

Keywords: a-priori domain decomposition, unstructured grid, Delaunay mesh generation

(14 pages, 2007)

111. O. lliev, R. Lazarov, J. Willems Numerical study of two-grid preconditioners for 1d elliptic problems with highly oscillating discontinuous coefficients Keywords: two-grid algorithm, oscillating coefficients, preconditioner

(20 pages, 2007)

112. L. Bonilla, T. Götz, A. Klar, N. Marheineke, R. Wegener

Hydrodynamic limit of the Fokker-Planckequation describing fiber lay-down processes

Keywords: stochastic dierential equations, FokkerPlanck equation, asymptotic expansion, Ornstein-

Uhlenbeck process

(17 pages, 2007)

\section{S. Rief}

Modeling and simulation of the pressing section of a paper machine

Keywords: paper machine, computational fluid dynamics, porous media

(41 pages, 2007)

114. R. Ciegis, O. Iliev, Z. Lakdawala

On parallel numerical algorithms for simulating industrial filtration problems

Keywords: Navier-Stokes-Brinkmann equations, finite volume discretization method, SIMPLE, parallel computing, data decomposition method

(24 pages, 2007)

\section{N. Marheineke, R. Wegener}

Dynamics of curved viscous fibers with surface tension

Keywords: Slender body theory, curved viscous bers with surface tension, free boundary value problem (25 pages, 2007)

116. S. Feth, J. Franke, M. Speckert Resampling-Methoden zur mse-Korrektur und Anwendungen in der Betriebsfestigkeit Keywords: Weibull, Bootstrap, Maximum-Likelihood, Betriebsfestigkeit

(16 pages, 2007)

117. H. Knaf

Kernel Fisher discriminant functions - a concise and rigorous introduction

Keywords: wild bootstrap test, texture classification, textile quality control, defect detection, kernel estimate, nonparametric regression

(30 pages, 2007)

118. O. Iliev, I. Rybak

On numerical upscaling for flows in heterogeneous porous media 
Keywords: numerical upscaling heterogeneous porous media, single phase flow, Darcy's law, multiscale problem, effective permeability, multipoint flux approximation, anisotropy

(17 pages, 2007)

\section{O. Iliev, I. Rybak}

On approximation property of multipoint flux approximation method

Keywords: Multipoint flux approximation, finite volume method, elliptic equation, discontinuous tensor coeffi-

cients, anisotropy

(15 pages, 2007)

120. O. Iliev, I. Rybak, J. Willems

On upscaling heat conductivity for a class of industrial problems

Keywords: Multiscale problems, effective heat conductivity, numerical upscaling, domain decomposition

(21 pages, 2007)

121. R. Ewing, O. Iliev, R. Lazarov, I. Rybak On two-level preconditioners for flow in porous media

Keywords: Multiscale problem, Darcy's law, single phase flow, anisotropic heterogeneous porous media, numerical upscaling, multigrid, domain decomposition, efficient preconditioner

(18 pages, 2007)

122. M. Brickenstein, A. Dreyer

POLYBORI: A Gröbner basis framework for Boolean polynomials

Keywords: Gröbner basis, formal verification, Boolean polynomials, algebraic cryptoanalysis, satisfiability

(23 pages, 2007)

\section{O. Wirjad}

Survey of $3 d$ image segmentation methods Keywords: image processing, 3d, image segmentation, binarization

(20 pages, 2007)

\section{S. Zeytun, A. Gupta}

A Comparative Study of the Vasicek and the CIR Model of the Short Rate

Keywords: interest rates, Vasicek model, CIR-model,

calibration, parameter estimation

(17 pages, 2007)

\section{G. Hanselmann, A. Sarishvili}

Heterogeneous redundancy in software quality prediction using a hybrid Bayesian approach

Keywords: reliability prediction, fault prediction, nonhomogeneous poisson process, Bayesian model averaging

(17 pages, 2007)

126. V. Maag, M. Berger, A. Winterfeld, K.-H Küfer

A novel non-linear approach to minimal area rectangular packing

Keywords: rectangular packing, non-overlapping constraints, non-linear optimization, regularization, relaxation

(18 pages, 2007)

127. M. Monz, K.-H. Küfer, T. Bortfeld, C. Thieke Pareto navigation - systematic multi-criteria-based IMRT treatment plan determination

Keywords: convex, interactive multi-objective optimization, intensity modulated radiotherapy planning

(15 pages, 2007)
128. M. Krause A Scherrer

On the role of modeling parameters in IMRT plan optimization

Keywords: intensity-modulated radiotherapy (IMRT) inverse IMRT planning, convex optimization, sensitivity analysis, elasticity, modeling parameters, equivalent uniform dose (EUD)

(18 pages, 2007)

\section{A. Wiegmann}

Computation of the permeability of porous materials from their microstructure by FFFStokes

Keywords: permeability, numerical homogenization

fast Stokes solver

(24 pages, 2007)

130. T. Melo, S. Nickel, F. Saldanha da Gama Facility Location and Supply Chain Management - A comprehensive review

Keywords: facility location, supply chain management network design

(54 pages, 2007)

131. T. Hanne, T. Melo, S. Nickel

Bringing robustness to patient flow management through optimized patient transports in hospitals

Keywords: Dial-a-Ride problem, online problem, case study, tabu search, hospital logistics

(23 pages, 2007)

132. R. Ewing, O. Iliev, R. Lazarov, I. Rybak, J. Willems

An efficient approach for upscaling properties of composite materials with high contrast of coefficients

Keywords: effective heat conductivity, permeability of fractured porous media, numerical upscaling, fibrous insulation materials, metal foams

(16 pages, 2008)

133. S. Gelareh, S. Nickel

New approaches to hub location problems in public transport planning Keywords: integer programming, hub location, transportation, decomposition, heuristic

(25 pages, 2008)

134. G. Thömmes, J. Becker, M. Junk, A. K. Vaikuntam, D. Kehrwald, A. Klar, K. Steiner, A. Wiegmann

A Lattice Boltzmann Method for immiscible multiphase flow simulations using the Level Set Method

Keywords: Lattice Boltzmann method, Level Set method, free surface, multiphase flow (28 pages, 2008

\section{J. Orlik}

Homogenization in elasto-plasticity

Keywords: multiscale structures, asymptotic homogenization, nonlinear energy

(40 pages, 2008)

136. J. Almquist, H. Schmidt, P. Lang, J. Deitmer, M. Jirstrand, D. Prätzel-Wolters, H. Becker

Determination of interaction between MCT1 and CAII via a mathematical and physiological approach

Keywords: mathematical modeling; model reduction: electrophysiology; $\mathrm{pH}$-sensitive microelectrodes; proton antenna

(20 pages, 2008)
137. E. Savenkov, H. Andrä, O. Iliev

An analysis of one regularization approach for solution of pure Neumann problem Keywords: pure Neumann problem, elasticity, regularization, finite element method, condition number (27 pages, 2008)

138. O. Berman, J. Kalcsics, D. Krass, S. Nickel The ordered gradual covering location problem on a network

Keywords: gradual covering, ordered median function, network location

(32 pages, 2008)

139. S. Gelareh, S. Nickel

Multi-period public transport design: A novel model and solution approaches Keywords: Integer programming, hub location, public transport, multi-period planning, heuristics (31 pages, 2008)

140. T. Melo, S. Nickel, F. Saldanha-da-Gama Network design decisions in supply chain planning

Keywords: supply chain design, integer programming models, location models, heuristics

(20 pages, 2008)

141. C. Lautensack, A. Särkkä, J. Freitag K. Schladitz

Anisotropy analysis of pressed point processes

Keywords: estimation of compression, isotropy test nearest neighbour distance, orientation analysis, polar ice, Ripley's K function

(35 pages, 2008)

142. O. Iliev, R. Lazarov, J. Willems

A Graph-Laplacian approach for calculating the effective thermal conductivity of complicated fiber geometries

Keywords: graph laplacian, effective heat conductivity, numerical upscaling, fibrous materials

(14 pages, 2008)

143. J. Linn, T. Stephan, J. Carlsson, R. Bohlin Fast simulation of quasistatic rod deformations for VR applications

Keywords: quasistatic deformations, geometrically exact rod models, variational formulation, energy minimization, finite differences, nonlinear conjugate gradients

(7 pages, 2008)

\section{J. Linn, T. Stephan}

Simulation of quasistatic deformations using discrete rod models

Keywords: quasistatic deformations, geometrically exact rod models, variational formulation, energy minimization, finite differences, nonlinear conjugate gradients

(9 pages, 2008

145. J. Marburger, N. Marheineke, R. Pinnau Adjoint based optimal control using meshless discretizations

Keywords: Mesh-less methods, particle methods, Eulerian-Lagrangian formulation, optimization strategies, adjoint method, hyperbolic equations

(14 pages, 2008

\section{S. Desmettre, J. Gould, A. Szimayer}

Own-company stockholding and work effort preferences of an unconstrained executive Keywords: optimal portfolio choice, executive compensation

(33 pages, 2008) 
147. M. Berger, M. Schröder, K.-H. Küfer A constraint programming approach for the two-dimensional rectangular packing problem with orthogonal orientations Keywords: rectangular packing, orthogonal orientations non-overlapping constraints, constraint propagation

(13 pages, 2008)

148. K. Schladitz, C. Redenbach, T. Sych, M. Godehardt

Microstructural characterisation of open foams using $3 d$ images

Keywords: virtual material design, image analysis, open foams

(30 pages, 2008)

149. E. Fernández, J. Kalcsics, S. Nickel, R. Ríos-Mercado

A novel territory design model arising in the implementation of the WEEE-Directive Keywords: heuristics, optimization, logistics, recycling (28 pages, 2008)

150. H. Lang, J. Linn

Lagrangian field theory in space-time for geometrically exact Cosserat rods Keywords: Cosserat rods, geometrically exact rods, small strain, large deformation, deformable bodies, Lagrangian field theory, variational calculus (19 pages, 2009)

151. K. Dreßler, M. Speckert, R. Müller, Ch. Weber

Customer loads correlation in truck engineering

Keywords: Customer distribution, safety critical components, quantile estimation, Monte-Carlo methods (11 pages, 2009)

152. H. Lang, K. Dreßler

An improved multiaxial stress-strain correction model for elastic FE postprocessing Keywords: Jiang's model of elastoplasticity, stress-strain correction, parameter identification, automatic differentiation, least-squares optimization, Coleman-Li algorithm

(6 pages, 2009)

153. J. Kalcsics, S. Nickel, M. Schröder A generic geometric approach to territory design and districting

Keywords: Territory design, districting, combinatorial optimization, heuristics, computational geometry (32 pages, 2009)

154. Th. Fütterer, A. Klar, R. Wegener An energy conserving numerical scheme for the dynamics of hyperelastic rods

Keywords: Cosserat rod, hyperealstic, energy conservation, finite differences

(16 pages, 2009)

155. A. Wiegmann, L. Cheng, E. Glatt, O. Iliev, S. Rief

Design of pleated filters by computer simulations

Keywords: Solid-gas separation, solid-liquid separation, pleated filter, design, simulation

(21 pages, 2009)

156. A. Klar, N. Marheineke, R. Wegener Hierarchy of mathematical models for production processes of technical textiles
Keywords: Fiber-fluid interaction, slender-body theory, turbulence modeling, model reduction, stochastic differential equations, Fokker-Planck equation, asymptotic expansions, parameter identification

(21 pages, 2009)

157. E. Glatt, S. Rief, A. Wiegmann, M. Knefel, E. Wegenke

Structure and pressure drop of real and virtual metal wire meshes

Keywords: metal wire mesh, structure simulation

model calibration, CFD simulation, pressure loss

(7 pages, 2009)

158. S. Kruse, M. Müller

Pricing American call options under the assumption of stochastic dividends - An application of the Korn-Rogers model

Keywords: option pricing, American options, dividends, dividend discount model, Black-Scholes model (22 pages, 2009)

159. H. Lang, J. Linn, M. Arnold

Multibody dynamics simulation of geometrically exact Cosserat rods

Keywords: flexible multibody dynamics, large deformations, finite rotations, constrained mechanical systems, structural dynamics

(20 pages, 2009)

160. P. Jung, S. Leyendecker, J. Linn, M. Ortiz Discrete Lagrangian mechanics and geometrically exact Cosserat rods

Keywords: special Cosserat rods, Lagrangian mechanics, Noether's theorem, discrete mechanics, frame-indifference, holonomic constraints

(14 pages, 2009)

161. M. Burger, K. Dreßler, A. Marquardt, M. Speckert

Calculating invariant loads for system simulation in vehicle engineering

Keywords: iterative learning control, optimal control

theory, differential algebraic equations(DAEs)

(18 pages, 2009)

162. M. Speckert, N. Ruf, K. Dreßler Undesired drift of multibody models excited by measured accelerations or forces Keywords: multibody simulation, full vehicle model, force-based simulation, drift due to noise

(19 pages, 2009)

163. A. Streit, K. Dreßler, M. Speckert, J. Lichter, T. Zenner, P. Bach

Anwendung statistischer Methoden zur Erstellung von Nutzungsprofilen für die Auslegung von Mobilbaggern

Keywords: Nutzungsvielfalt, Kundenbeanspruchung, Bemessungsgrundlagen

(13 pages, 2009)

164. I. Correia, S. Nickel, F. Saldanha-da-Gama Anwendung statistischer Methoden zur Erstellung von Nutzungsprofilen für die Auslegung von Mobilbaggern

Keywords: Capacitated Hub Location, MIP formulations (10 pages, 2009)

165. F. Yaneva, T. Grebe, A. Scherrer

An alternative view on global radiotherapy optimization problems

Keywords: radiotherapy planning, path-connected sublevelsets, modified gradient projection method, improving and feasible directions

(14 pages, 2009)
166. J. I. Serna, M. Monz, K.-H. Küfer, C. Thieke Trade-off bounds and their effect in multicriteria IMRT planning

Keywords: trade-off bounds, multi-criteria optimization, IMRT, Pareto surface

(15 pages, 2009)

167. W. Arne, N. Marheineke, A. Meister, R. Wegener

Numerical analysis of Cosserat rod and string models for viscous jets in rotational spinning processes

Keywords: Rotational spinning process, curved viscous fibers, asymptotic Cosserat models, boundary value problem, existence of numerical solutions

(18 pages, 2009)

168. T. Melo, S. Nickel, F. Saldanha-da-Gama An LP-rounding heuristic to solve a multiperiod facility relocation problem Keywords: supply chain design, heuristic, linear programming, rounding

(37 pages, 2009)

169. I. Correia, S. Nickel, F. Saldanha-da-Gama Single-allocation hub location problems with capacity choices

Keywords: hub location, capacity decisions, MILP formulations

(27 pages, 2009)

170. S. Acar, K. Natcheva-Acar A guide on the implementation of the Heath-Jarrow-Morton Two-Factor Gaussian Short Rate Model (HJM-G2++)

Keywords: short rate model, two factor Gaussian,

G2++, option pricing, calibration

(30 pages, 2009)

171. A. Szimayer, G. Dimitroff, S. Lorenz A parsimonious multi-asset Heston model: calibration and derivative pricing Keywords: Heston model, multi-asset, option pricing, calibration, correlation

(28 pages, 2009)

Status quo: July 2009 